\title{
Internal gravity waves in a dipolar wind: a wave-vortex interaction experiment in a stratified fluid
}

\author{
By RAMIRO GODOY-DIANA ${ }^{1,2}$, JEAN-MARC CHOMAZ \\ AND CLAIRE DONNADIEU ${ }^{1}$ \\ ${ }^{1}$ LadHyX, CNRS-École Polytechnique, F-91128 Palaiseau Cedex, France \\ ${ }^{2}$ LOCEAN, Université Pierre et Marie Curie-CNRS-IPSL, Tour 45-55, 4ème étage, \\ Boîte 100, 4 place Jussieu 75252 Paris Cedex 05, France \\ ramiro.godoy-diana@lodyc.jussieu.fr
}

(Received 15 February 2005 and in revised form 25 July 2005)

An experimental study on the interaction of the internal wave field generated by oscillating cylinders in a stratified fluid with a pancake dipole is presented. The experiments are carried out in a salt-stratified water tank with constant Brunt-Väisälä frequency $(N)$. Experimental observations of the deformation of the wave beams owing to the interaction with the dipole are presented. When the wave and the dipole propagate horizontally in opposite directions (counterpropagating case), the phase line of the gravity wave beam steepens towards the vertical as it enters the dipolar field and it may even reach a turning point where the wave is reflected. When the dipole and the wave propagate in the same direction (copropagating case), the wave beam is bent towards the horizontal and may be absorbed by the dipole. These observations are in good agreement with a two-dimensional ray-theoretic model, even if the flow is fully three-dimensional and, the vertical shear induced by the dipole being too strong, the hypothesis of slow variation assumed in the WKB approximation is not verified. When the waves encounter a critical layer, we show by rigorous measurement that momentum is transferred to the dipole. New three-dimensional effects of the dipolar velocity field on the propagating internal waves are also discussed. In particular, focusing and refraction of a wave beam occurring because of the horizontal structure of the background dipolar flow allow us to explain some of the observed features that cannot be accounted for through the two-dimensional ray theory.

\section{Introduction}

Stably stratified fluids are often encountered in the atmosphere and ocean. Owing to the inhibition of vertical motions, they give rise to layered flows that can be described by potential vorticity modes (Riley, Metcalfe \& Weissman 1981; Lilly 1983; Riley \& Lelong 2000). On the other hand, the restoring force that flattens isopycnal surfaces also allows the propagation of internal gravity waves. The time scales relevant to these two types of motion separate when the stratification is strong; internal waves evolve on a fast time scale based on the buoyancy frequency $\left(T_{N}=N^{-1}\right)$ and are associated with zero potential vorticity, whereas the quasi-horizontal motions that possess potential vorticity (PV modes, using the terminology of Riley \& Lelong 2000) evolve on a slower time scale in terms of the horizontal advection $\left(T_{A}=L_{h} / U\right.$, where $L_{h}$ and $U$ are the characteristic horizontal length and velocity scales). An illustration 
of the difference between these two modes can be observed when the motion is initially confined to a particular region of space; as vertical motions are strongly constrained, energy is either radiated as internal waves, which propagate away from the initially turbulent region, or transferred to horizontal advective motions which are eventually organized as quasi-two-dimensional vortices. The creation of these patches of potential vorticity has been widely observed in laboratory experiments (e.g. Lin \& Pao 1979; Bonneton, Chomaz \& Hopfinger 1993; Fincham, Maxworthy \& Spedding 1996) and numerical simulations (e.g. Métais \& Herring 1989; Majda \& Grote 1997). The so-called pancake vortices appearing late in the evolution of stratified flows have also been intensively studied (Flór \& van Heijst 1996; Spedding, Browand \& Fincham 1996; Bonnier, Eiff \& Bonneton 2000; Beckers et al. 2001; Godoy-Diana \& Chomaz 2003).

The horizontal Froude number $F_{h}=U / N L_{h}$ compares the horizontal advection time scale $L_{h} / U$ to the Brunt-Väisälä frequency. When $F_{h}$ is small, the theory first proposed by Riley et al. (1981) predicts no interaction at leading order between the wave modes and the PV modes. Because of the time-scale separation, the interaction between internal gravity waves and PV modes has usually been studied as a multiplescale problem (the wave phase varies on a fast time scale while its amplitude and the PV mode depend only on a slow time scale) and weakly nonlinear interactions have been predicted theoretically (see e.g. Riley \& Lelong 2000). These are resonant triad interactions as those first studied by Phillips (1966) for internal waves, but involving one or two PV modes in the triad. In the case of a single PV mode and two wave modes, no wave-PV transfer is predicted and the PV mode only provides a way for the energy exchange between the two wave modes (Lelong \& Riley 1991; Godeferd \& Cambon 1994), on the contrary, when the triad is formed by one wave mode and two PV modes, a near-resonant PV-wave transfer can be expected (Bartello 1995). Additionally, PV modes can act as a source of internal waves through adjustment of unbalanced isopycnal surfaces to an equilibrium state (see e.g. Beckers et al. (2001) for numerical simulations of the wave emission by an unbalanced monopole and Afanasyev (2003) for experimental results of the emission by an adjusting dipole). An unbalanced vortex can be treated as an initial disturbance to the stratified fluid and the internal wave emission described as in Lighthill (1996). The permanent internal wave emission by a balanced but elliptical vortex has been analysed as a radiation problem by Plougonven \& Zeitlin (2002) allowing for changes in the source vortex induced by wave radiation.

Internal waves are known to have a fundamental role in the momentum transfers in the atmospheres and, through turbulence induced by wave breaking, in the diapycnal mixing in the oceans (see reviews by Staquet \& Sommeria 2002; Fritts \& Alexander 2003). The internal wave energy transfer to PV modes has been invoked thinking of breaking internal waves which may cause strong mixing and result in a threedimensional turbulent region from which a PV component can emerge (Staquet, Bouruet-Aubertot \& Koudella 2001; Lelong \& Sundermeyer 2005). These dissipative mechanisms are generally the only wave-mean flow interaction considered in the gravity-wave parameterizations used in global atmospheric circulation models, assuming that other interactions occurring without wave breaking (or other dissipative mechanism allowing a suitable representation as a mean force) are not significant. Bühler \& McIntyre $(1998,2003)$ put a question mark on these assumptions through the analysis of model examples showing that cumulative deformation of PV components (the mean flow) owing to non-dissipative gravity waves may occur and name this reaction effect to wave refraction a remote recoil. 


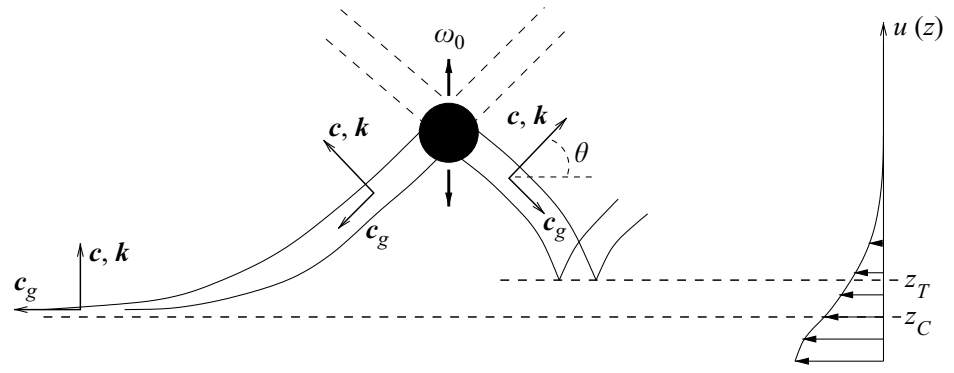

FIGURE 1. Schematic diagram of the internal waves generated by an oscillating cylinder in a sheared flow. Critical points are reached at $z_{C}$ and $z_{T}$.

The effect of a background mean flow on the waves is usually understood by referring to the Doppler shifting of the wave frequency. The Doppler shift of waves owing to a shear flow has been the subject of many theoretical studies (e.g. Bretherton 1966; Booker \& Bretherton 1967), laboratory experiments (e.g. Koop 1981; Thorpe 1981) and numerical simulations (e.g. Winters \& D'Asaro 1994; Javam, Imberger \& Armfield 2000; Sutherland 2000; Staquet \& Huerre 2002; Edwards \& Staquet 2005). The case where the background shear is a vortical flow has also been studied by Moulin (2003); Moulin \& Flór (2004, 2005) experimentally and theoretically using a ray-tracing code that describes the wave distortion by a vortex. According to linear theory, critical levels exist where the shifted frequency reaches one of the cutoff values imposed by the stratification ( 0 and $N$ in the absence of rotation) and the waves are either absorbed by the mean flow in a critical layer or reflected at a turning point (figure 1). In the linear analysis of Booker \& Bretherton (1967), plane internal waves of small amplitude are completely absorbed by the mean flow in the vicinity of a critical layer. They showed an exponential attenuation of the waves across the critical layer of order $\exp \left[-2 \pi(R i-1 / 4)^{1 / 2}\right]$ in terms of the local Richardson number $R i=N^{2} /|\mathrm{d} \boldsymbol{u} / \mathrm{d} z|^{2}$, meaning that, for $R i>1 / 4$, no part of the incoming wave is transmitted through the critical layer and all the wave energy is transferred to the mean flow. The linear inviscid prediction of complete absorption at the critical level may not be fully accomplished in real flows because finite-amplitude effects may lead to a partial reflection (e.g. Winters \& D'Asaro 1994) or to wave breaking (McIntyre 2000). In reality, spatially localized wave packets should be considered instead of plane waves. As a result, the dispersion of the different components in a wave packet induces a spreading of the critical levels (see e.g. McIntyre 2000) and some waves are transmitted carrying a significant fraction of the incoming wave energy through the critical layer (Javam \& Redekopp 1998). A three-dimensional background shear may also reduce the magnitude of critical-level effects with respect to the predictions of the usual models that consider unidirectional parallel shear (Staquet \& Sommeria 2002). Additionally, as pointed out by Broutman et al. (1997), the time-dependence of the background shear may change significantly the Doppler spreading of internal waves observed in a stationary shear.

In this paper, we study experimentally the interaction between the internal wave field produced by an oscillating cylinder in a strongly stratified fluid and a pancake dipole, representing a prototype PV mode. The observations are first interpreted in terms of two-dimensional ray theory for waves in a shear flow (e.g. Bretherton 1966). The internal wave beams are refracted by the dipole (which constitutes the horizontal background flow) and the existence of critical levels for the wave propagation is 
verified by the experiments. A particle image velocimetry (PIV) measurement setup gives access to the velocity vector fields in vertical $(x, z)$ and horizontal $(x, y)$ planes and permits the calculation of the theoretical predictions for the critical levels using experimental data. The linear inviscid Wentzel-Kramer-Brillovin (WKB) approximation at the base of ray theory assumes waves propagating in a slowly varying background flow (meaning that variations occur on much larger length and time scales than the wavelength and wave period). This scale separation assumption implies that, at leading order, the dispersion relation for internal waves in a steady medium is locally valid. Although the assumption of weak variation is not verified for the vertically sheared flow produced by the pancake dipole of the present experiments, especially regarding the ratio of wavelength to vertical length scale of the vortical flow, which are of comparable order, the location of a critical layer and a turning point observed in the experiments are predicted surprisingly accurately by the two-dimensional raytheoretical model. The two-dimensional model considered is strictly valid only in the vertical symmetry plane $(x, z)$ of the dipolar field, where the dipole induces motion only in the $x$-direction and the internal gravity wave vector is always in that plane. Because of the three-dimensional nature of the dipole field, additional features are observed in the wave propagation which cannot be explained by the two-dimensional model. The initially straight phase planes of the waves (which are generated by a cylinder with axis in the $y$-direction, i.e. orthogonal to the two-dimensional plane used for the ray model) are deformed in the horizontal planes by the dipole. Refraction and focusing effects, which are inherently three-dimensional, occur on the internal wave beams. A defocusing effect is observed when the wave and the dipole propagate horizontally in the same direction and it may limit the extent of wave-vortex momentum transfer due to critical-layer type interactions in the present experiments.

The present paper is organized as follows. A brief review of the linear theory of internal gravity waves is presented in $\S 2$. The experimental setup is described in $\S 3$ followed by the presentation of the basic states for the experimental waves and dipoles in $\S 4$. Section 5 is devoted to the observations and discussion. Concluding remarks appear in $\S 6$.

\section{Linear theory for internal waves}

In this section, we review briefly some standard results of the linear theory for internal gravity waves in a continuously stratified fluid with a background sheared flow as discussed by Lighthill (1978) and Gill (1982). Consider sinusoidal waves of frequency $\omega$ and wavevector $\boldsymbol{k}=\left(k_{x}, k_{y}, k_{z}\right)$ such that all fields associated to their motion can be written as $\propto \exp [-\mathrm{i}(\boldsymbol{k} \cdot \boldsymbol{x}-\omega t)]$. The well-known dispersion relation for internal waves in a medium at rest of constant buoyancy frequency $N$,

$$
\omega^{2}=N^{2} \cos ^{2} \theta=N^{2} \frac{k_{x}^{2}+k_{y}^{2}}{k^{2}},
$$

where $\theta$ is the angle of the wavevector $\boldsymbol{k}$ with respect to the horizontal and $k=|\boldsymbol{k}|$, is obtained from the Boussinesq equations linearized about a background hydrostatic equilibrium. We remark that the phase velocity $\boldsymbol{c}=\left(\omega / k^{2}\right) \boldsymbol{k}$ is orthogonal to the group velocity $c_{g}=\nabla_{k} \omega$, and energy therefore propagates perpendicular to the wavevector, i.e. in the phase plane. An energy flux vector can be written as

$$
\boldsymbol{I}=E \boldsymbol{c}_{g},
$$

in terms of the wave energy density $E$ and the group velocity. 
When $N$ is not constant or a background shear $\boldsymbol{U}(\boldsymbol{x}, t)$ is considered, the WKB approximation can be used if $N(\boldsymbol{x}, t)$ and $\boldsymbol{U}(\boldsymbol{x}, t)$ vary on a time scale and length scale much larger than the wave period and wavelength, respectively. In such case, the waves obey locally the dispersion relation (2.1) in the reference frame moving with the fluid. If we now think of waves propagating through a mean flow $\boldsymbol{U}(\boldsymbol{x}, t)$, the absolute wave frequency $\omega_{a}$ (measured in the fixed frame of reference) is Dopplershifted with respect to the relative frequency $\omega_{r}$ (measured in the frame moving with the background velocity) according to

$$
\omega_{a}=\omega_{r}+\boldsymbol{k} \cdot \boldsymbol{U},
$$

where $\omega_{r}$ is given by equation (2.1) substituting $\omega$ by $\omega_{r}$, pointing out the fact that in the frame of reference moving with the fluid, the waves are dispersed in the same manner as in a fluid at rest. Similarly, the absolute group velocity in the fixed frame is $\boldsymbol{c}_{g a}=\boldsymbol{c}_{g r}+\boldsymbol{U}$, where $\boldsymbol{c}_{g r}$ is the group velocity in the frame moving with the mean flow. It should be noted that the absolute phase velocity $\boldsymbol{c}_{a}=\boldsymbol{c}_{r}+(\boldsymbol{k} \cdot \boldsymbol{U}) \boldsymbol{k} / k^{2}$, where $\boldsymbol{c}_{r}$ is the phase velocity in the frame moving with the fluid, is no more orthogonal to the absolute group velocity. The phase velocity does not transform as a usual vector in a change of reference frame because it characterizes only the apparent displacement of isophase surfaces and should therefore stay normal to the isophases in any frame.

Because of the non-homogeneity, the wave energy that propagates at the group velocity $c_{g}$ is refracted following paths usually known as rays. These rays can be traced in the absolute frame of reference by a position vector $\boldsymbol{x}$ that moves with the absolute group velocity, i.e. obeying

$$
\frac{\mathrm{d} \boldsymbol{x}}{\mathrm{d} t}=\boldsymbol{c}_{g a}=\boldsymbol{U}+\boldsymbol{c}_{g r},
$$

while the changes in the wavevector along these rays are given by (see Lighthill 1978, for details)

$$
\frac{\mathrm{d} \boldsymbol{k}}{\mathrm{d} t}=-\nabla \omega_{a}=-\nabla(\boldsymbol{k} \cdot \boldsymbol{U})-\nabla \omega_{r}
$$

In this equation the time derivative should be considered along a ray and is equivalent to the rate of change with time at a position that moves with the group velocity, i.e. $\mathrm{d} / \mathrm{d} t=\partial / \partial t+\left(\boldsymbol{c}_{g a} \cdot \nabla\right)$. Using these ray tracing equations (2.4) and (2.5), we can show that the time derivative of $\omega_{a}=\omega_{a}(\boldsymbol{k}, \boldsymbol{x})$ is zero, i.e. that the frequency is constant along a ray. Because $\omega_{a}$ remains constant, any changes in $\boldsymbol{k} \cdot \boldsymbol{U}$ are accompanied according to equation (2.3) by a change in $\omega_{r}$ which in turn modifies the wave vector direction according to equation (2.1). Equation (2.4) states that the wave propagates with the absolute group velocity equal to the sum of the mean flow velocity vector and the relative group velocity. In addition to the evolution of the wavevector due to changes in the local dispersion relation (i.e. changes in $N$ ), second term in equation (2.5), we must add the changes due to the background shear, first term in equation (2.5).

In contrast to the absolute frequency, the frequency in the moving frame $\omega_{r}$ is not conserved, nor is the mean wave energy along a ray $E_{r}$ (because energy can be exchanged between the waves and the mean flow). A useful conserved quantity along rays is the wave action $\mathscr{A}_{r}=E_{r} / \omega_{r}$, which obeys:

$$
\frac{\partial \mathscr{A}_{r}}{\partial t}+\nabla \cdot\left[\left(\boldsymbol{U}+\boldsymbol{c}_{g r}\right) \mathscr{A}_{r}\right]=0 .
$$

The conservation of wave action determines a synchronized behaviour of the relative frequency and wave energy density: $E_{r}$ increases (decreases) when the ray passes 
through a region of higher (lower) $\omega_{r}$. The change in wave energy is provided (absorbed) by the mean flow. Equation (2.6) is simplified for waves of fixed frequency and for a steady base state, corresponding to the approximation that we will make throughout this paper, giving

$$
\nabla \cdot\left[\left(\boldsymbol{U}+\boldsymbol{c}_{g r}\right) \mathscr{A}_{r}\right]=0 .
$$

This means that the flow of wave action along a ray tube is constant, i.e. that the magnitude of the wave action flux $\left(\boldsymbol{U}+\boldsymbol{c}_{g r}\right) \mathscr{A}_{r}$ changes along a ray tube in inverse proportion to the area of its cross-section.

\subsection{Critical levels for two-dimensional waves}

Two limit cases of particular interest can be illustrated considering the simple case of waves in a vertically sheared horizontal background flow $\boldsymbol{U}=\boldsymbol{U}(z)$ and linear background stratification, that is, of constant $N$. Together with $\omega_{a}$, in such a configuration $k_{x}$ and $k_{y}$ are also constant along a ray since the flow is invariant by translation in time and in the $x$ and $y$ directions. In that case, the Doppler shift of $\omega_{r}$ can be determined solely by equation (2.3) which can be rewritten as $\omega_{r}=\omega_{a}-\boldsymbol{k}_{h} \cdot \boldsymbol{U}$, where $\boldsymbol{k}_{h} \equiv\left(k_{x}, k_{y}, 0\right)$ is the horizontal wavevector. If $\boldsymbol{k}_{h}$ and $\boldsymbol{U}$ point in opposite directions, equation (2.3) determines that an increase in $\boldsymbol{k}_{h} \cdot \boldsymbol{U}$ increases the intrinsic frequency $\omega_{r}$. Using the relative dispersion relation (2.1), this results in a decrease of $\theta$, i.e. a tilt towards the horizontal of the wavevector $\boldsymbol{k}$. A turning point exists at $z=z_{T}$ where

$$
\omega_{r}=\omega+\left|\boldsymbol{k}_{h} \cdot \boldsymbol{U}\left(z_{T}\right)\right|=N
$$

(and $k_{z}=0$ ) since waves are not allowed by the stratification beyond that point and the wave beam is reflected. The other limit case appears when $\boldsymbol{k}_{h}$ points to the same direction of $\boldsymbol{U}$. A critical layer exists at $z=z_{C}$ where $\omega_{r}$ vanishes:

$$
\omega_{r}=\omega-\left|\boldsymbol{k}_{h} \cdot \boldsymbol{U}\left(z_{C}\right)\right| \rightarrow 0
$$

and, $\boldsymbol{k}_{h}$ being constant, $k_{z} \rightarrow \infty$ as $\theta \rightarrow \pi / 2$. The linear inviscid analysis is singular at this point, but, relaxing the WKB approximation, the wave energy at the critical level is found to be attenuated and an energy transfer from the wave to the mean flow is predicted (Booker \& Bretherton 1967). This is actually a consequence of the conservation of the wave action, which implies that where $\omega_{r} \rightarrow 0$ all the wave energy is lost to the mean flow.

Both cases are illustrated schematically in figure 1, where the beams emanating from an oscillating horizontal cylinder in a vertically sheared background flow are represented (see also Koop 1981). The time evolution of the vertical component of the wavevector is obtained from equation (2.5), it reads:

$$
\frac{\mathrm{d} k_{z}}{\mathrm{~d} t}=-\boldsymbol{k}_{h} \cdot \frac{\partial \boldsymbol{U}}{\partial z} .
$$

This equation shows that changes in $k_{z}$ are linear with time, which means that the turning point where $k_{z}=0$ can be reached in a finite time whereas the time to reach the critical layer where $k_{z} \rightarrow \infty$ is infinite. The latter means that rays are asymptotic to the critical layer. The amplitude variations can be inferred from equation (2.7) for the wave action flux. Together with the fact that sections of a ray tube by each horizontal plane have the same area - because $\boldsymbol{k}_{h}$ is constant along a ray (Lighthill 1978 ) - this equation implies that the vertical component of the wave action flux is also constant along a ray. For the internal waves of the present case, using (2.1) and the third component of the group velocity (i.e. $\partial \omega_{r} / \partial k_{z}$ ), this vertical component of 


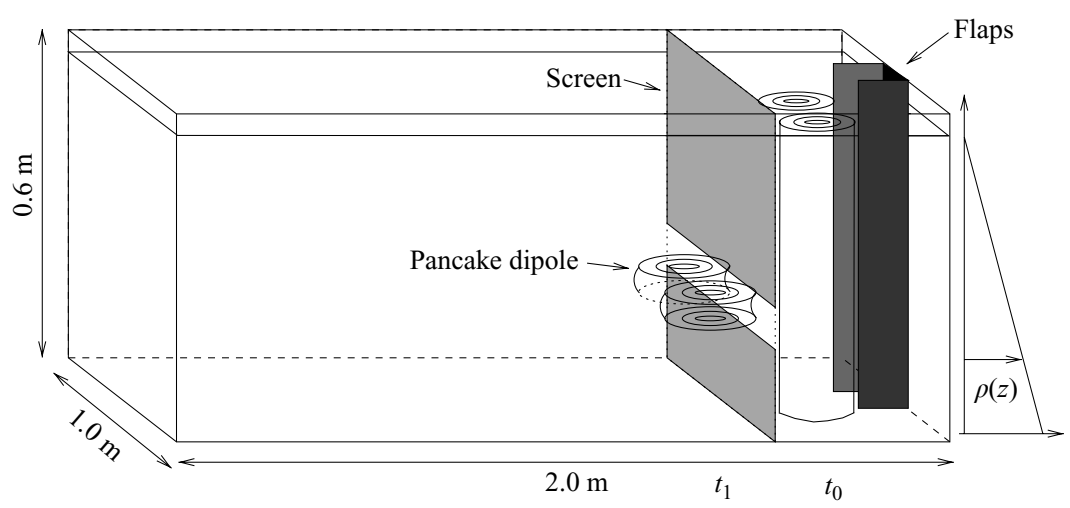

FIGURE 2. Experimental set-up used to produce a pancake dipole. At time $t_{0}$, a columnar vortex pair is created by closing the vertical flaps on the right-hand side. At time $t_{1}$, the pancake dipole emerges from the open region of the screen. Dimensions of the tank and a qualitative plot of the linear density gradient are also shown. (From Godoy-Diana et al. 2004)

the wave action flux $\mathscr{A}_{r} \partial \omega_{r} / \partial k_{z}$ is found to be proportional to $W^{2} \tan \theta$, where $W$ is the amplitude of the vertical velocity (and hence $E_{r} \propto W^{2}$ ). This result implies that wave amplitudes diminish when the rays approach the critical layer, whereas they are increased on bending towards the vertical prior to reaching the turning point.

\section{Experimental set-up}

The experiments are conducted in a salt water tank of $1 \mathrm{~m} \times 2 \mathrm{~m}$ base and $0.6 \mathrm{~m}$ height filled with a linear stratification. Internal waves are generated by means of either one or an array of oscillating horizontal cylinders, which can be placed at different heights and horizontal positions. For frequencies below $N$, each cylinder generates the well-known St Andrew's Cross pattern of wave beams (see e.g. Mowbray \& Rarity 1967) with directions of propagation depending on the oscillation frequency of the cylinders $\omega$ according to the dispersion relation (2.1), $\cos \theta=\omega / N$. The width of each wave beam is comparable to the source size (i.e. the cylinder diameter) and grows very slowly with distance from the source, whereas the amplitude of the wave motions is determined by the amplitude of the cylinder oscillations (see e.g. Sutherland et al. 1999, for a detailed study of the structure of the internal wave beams generated by a vertically oscillating cylinder).

A pancake dipole is generated in two steps as shown schematically in figure 2 (Godoy-Diana, Chomaz \& Billant 2004): first, a pair of vertical flaps placed on one side of the tank and spanning its whole height close to form a columnar dipole (Billant \& Chomaz 2000). The upper and lower layers of the initial dipole are blocked by a vertical screen placed perpendicularly to its moving path. The screen acts as a diaphragm which allows the evolution of only a horizontal slice of the original dipole. This method renders repeatable structures and ensures a unique propagation direction of the dipole, an advantage over the usual set-ups where the path followed by dipoles generated after the collapse of an initially turbulent jet is less predictable. Three nondimensional control parameters can be defined for the dipole coming out of the screen: the Reynolds number $R e_{0}=U_{0} L_{h 0} / v$, the horizontal Froude number $F_{h 0}=U_{0} / N L_{h 0}$ and the aspect ratio $\alpha_{0}=L_{v 0} / L_{h 0}$, where $U_{0}, L_{h 0}$ and $L_{v 0}$ are, respectively, the initial translation speed and the horizontal and vertical length scales, and $v$ is the kinematic 
viscosity. In practice, we define the horizontal length scale $L_{h 0}$ as the dipole radius, which is initially determined by the size of the flaps. The dipole translation speed $U_{0}$ can be controlled by the closing speed and final angle of the flaps while the initial vertical length scale $L_{v 0}$ is determined by the height of the gap on the screen. For the present experiments these parameters were kept within the ranges of $F_{h 0}=0.06-$ $0.18, R e_{0}=131-182$ and $\alpha_{0}=0.4-1.2$. All observations are made after the dipole has crossed the diaphragm, so $t=0$ is defined when the maximum velocity region at the core of the dipole is out of the screen $\left(t_{1}\right.$ in figure 2 , defined as $30 \mathrm{~s}$ after the closing the flaps).

Two different configurations were used in order to allow for interaction of the dipole with: $(a)$ waves with horizontal component of the wave vector $k_{x}$ pointing in the opposite direction of the translation velocity of the dipole (counterpropagating case, figure $3 a$ ) and $(b)$ with $k_{x}$ pointing in the same direction (copropagating case, figure $3 b$ ). Only one cylinder is depicted in these schematic diagrams for clarity. In the actual set-up of case $(a)$ an array of three identical cylinders separated horizontally from each other by a distance of six times their diameter was used to create a more extended wave field (figure $3 c$ ). In case $(b)$, a single cylinder was used because of the lack of space owing to the presence of the screen. Several beams are nonetheless present in the test section due to the multiple reflections on the screen and the free surface (figure $3 d$ ).

A two-dimensional particle image velocimetry (PIV) system (FlowMaster 3S manufactured by La Vision) was used to measure the velocity field. The image acquisition is made by a double-frame camera with resolution of $1280 \times 1024$ pixels and a 12-bit dynamic range. The light flashes were generated by chopping a continuous beam $5 \mathrm{~W}$ argon laser with an optoacoustic switch. The laser beam was spread into a sheet by an array of cylindrical lenses at the end of an optic fibre. The thickness of the light sheet was approximately $5 \mathrm{~mm}$ at the region of interest. Two measurement set-ups were used in order to look at vertical and horizontal planes. The vertical plane was aligned with the propagation direction of the dipole, passing through the velocity maximum and cutting the dipole symmetrically into two halves. The horizontal plane was placed at the position of the maximum velocity, i.e. at the midplane. In both cases, titanium dioxide $\left(\mathrm{TiO}_{2}\right)$ particles are used as flow seeding (sizes $\sim 1-100 \mu \mathrm{m}$ ). These particles are slightly heavier than the salt-water at the bottom of the stratification, but they sediment very slowly (in several hours) so that, for the time scales used for the PIV shots, they can be reasonably regarded as neutrally buoyant. The wide variation in particle size of the $\mathrm{TiO}_{2}$ powder allows particles to be uniformly distributed throughout the whole height of the experimental tank. The choice of the optimal time $(\Delta t)$ between each pair of images used for calculating the correlation and from that the velocity field, had to be done carefully since the characteristic velocities associated to the waves and the vortex differ considerably (from $\sim 10^{-3} \mathrm{~m} \mathrm{~s}^{-1}$ for the wave motions to $\sim 10^{-2} \mathrm{~m} \mathrm{~s}^{-1}$ for the initial dipole). Two acquisition schemes were used in order to have an appropriate resolution for both velocity scales. Most image series were taken using a single-frame mode and $\Delta t=200$ and $250 \mathrm{~ms}$, allowing for the identification of the internal wave velocity field. Additionally, double-frame sequences with $\Delta t=50$ and $100 \mathrm{~ms}$ were used in order to measure the dipole velocity field at the initial stages which could not be resolved with the former acquisition scheme. The need for such different schemes is illustrated in figure 4, where the velocity field of the dipole at $t=0$ on the vertical symmetry plane is shown as measured with acquisitions where $\Delta t=50 \mathrm{~ms}$ (figure $4 a$ ) and $\Delta t=500 \mathrm{~ms}$ (figure $4 b$ ). In the former, the dipole field is fully recovered, but no waves can be seen, whereas in the latter no 
(a)

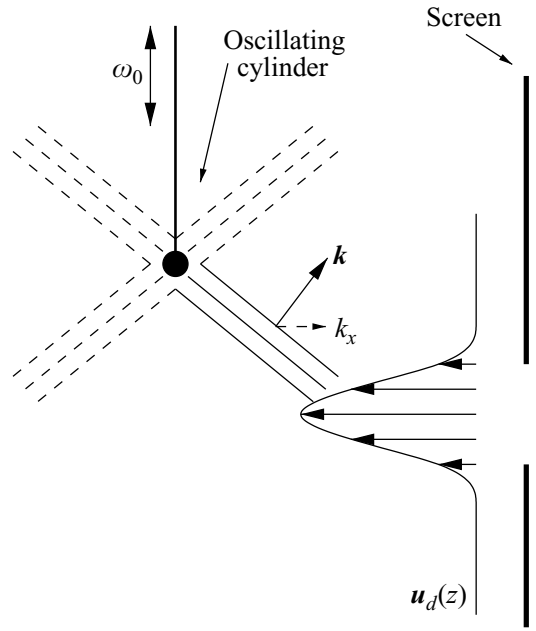

(b)

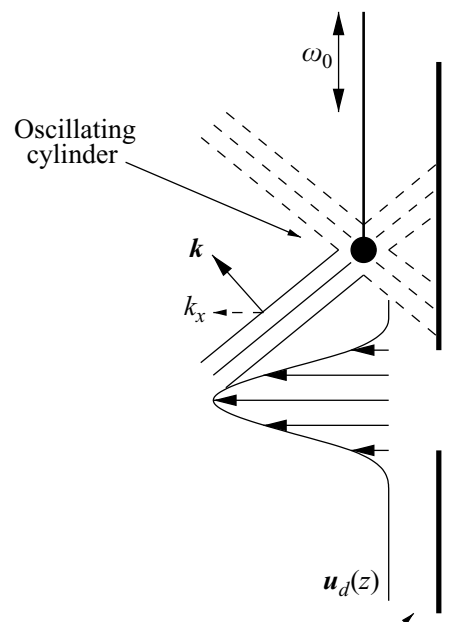

(c)

(d)

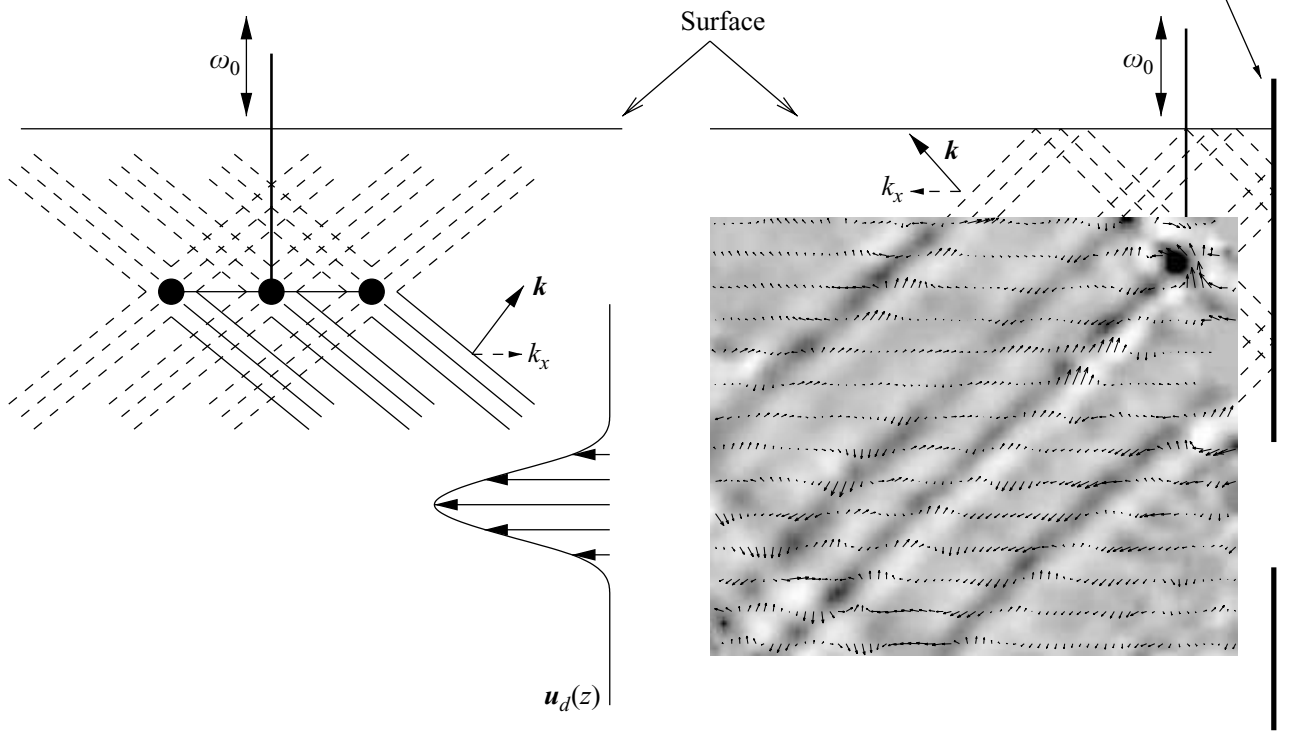

FIGURE 3. $(a, b)$ Schematic diagrams of the position of the oscillating cylinder with respect to the path of the dipole. Two cases are shown where the horizontal projection of the wavevector $k_{x}$ of the relevant ray and the translation direction of the dipole $\boldsymbol{u}_{d}$ are $(a)$ antiparallel and (b) parallel. (A side view of the vertical symmetry plane of the dipole is shown where $\boldsymbol{u}_{d}$ represents a profile of the dipole velocity.) (c) Diagram of the actual set-up used with an array of three identical cylinders. $(d)$ PIV measurements showing the horizontal vorticity field in grey scale and the velocity vectors as arrows on a vertical plane of the internal wave pattern after reflection of the direct beams of a single cylinder on the screen and the free surface. The paths followed by the reflected beams are drawn schematically.

valid correlation peak could be calculated at the centre of the dipole, but the internal wave pattern produced by the adjustment of the initial dipole is clearly retrieved.

\section{Basic states}

The initial state for the pancake dipole after it has come out of the diaphragm entirely is presented in figure 5. Velocity vector fields calculated from PIV measurements 
(a)

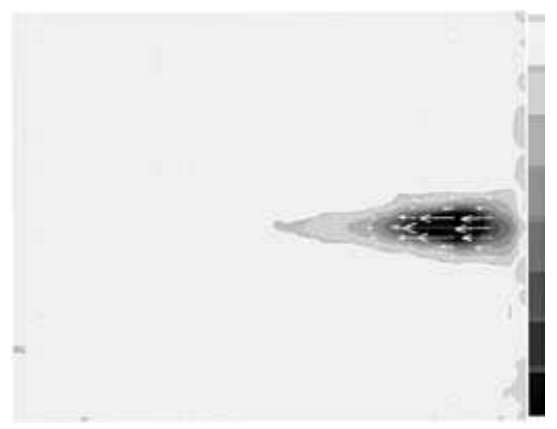

(b)

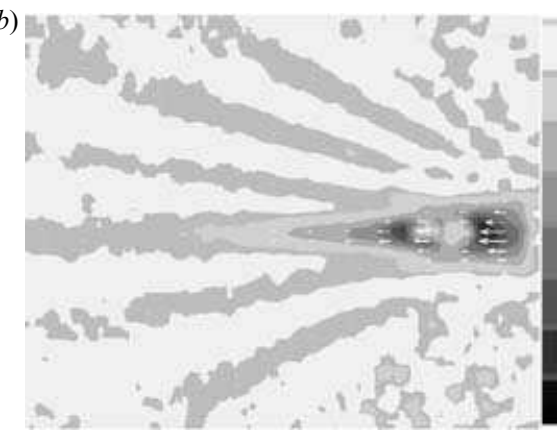

FIGURE 4. Horizontal velocity field at the vertical symmetry plane of the dipole at $t=0$ calculated from PIV images with $(a) \Delta t=50 \mathrm{~ms}$ and $(b) \Delta t=250 \mathrm{~ms}$. The field of view in the $(x, z)$-plane is $49 \times 39 \mathrm{~cm}^{2}$. The velocity grey scale goes from $0.001 \mathrm{~m} \mathrm{~s}^{-1}$ to $-0.01 \mathrm{~m} \mathrm{~s}^{-1}$.

(a)

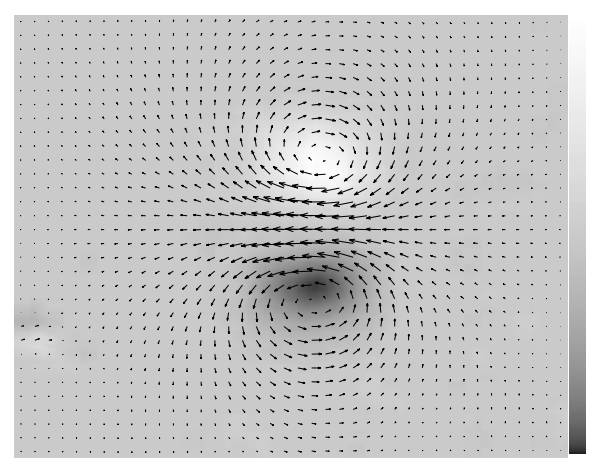

(b)

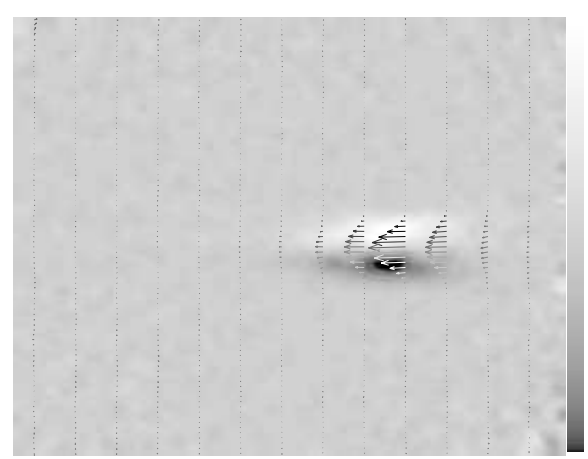

FIGURE 5. Velocity vector fields calculated from PIV measurements in the $(a)$ horizontal midplane and the $(b)$ vertical symmetry plane passing through the moving direction of the dipole. $(a)$ Vertical and $(b)$ horizontal vorticity fields are shown as background images. Fields of view and greyscales: (a) $31 \times 24 \mathrm{~cm}^{2}$ in the $(x, y)$-plane and a $\pm 1 \mathrm{~s}^{-1}$ grey scale; (b) $49 \times 39 \mathrm{~cm}^{2}$ in the $(x, z)$-plane and $\mathrm{a} \pm 0.5 \mathrm{~s}^{-1}$ grey scale.

in the horizontal midplane and the vertical symmetry plane passing through the moving direction of the dipole are shown. The calculated vorticity fields are also shown as background images. The horizontal structure of the dipole closely resembles a Lamb-Chaplygin dipole. Dipolar vortices in stratified fluids were compared for the first time with this theoretical model by van Heijst \& Flór (1989) and Flór \& van Heijst (1994). The Lamb-Chaplygin dipole also modelled well the horizontal structure of the columnar vortex pair studied by Billant \& Chomaz (2000). The vertical structure can be fitted accurately by a Gaussian variation. The vertical and horizontal characteristic length scales that can be defined from these images (e.g. the dipole radius for the horizontal and the Gaussian half-width for the vertical) grow slowly as the dipole decays by the action of viscosity (see Flór, van Heijst \& Delfos 1995; Beckers et al. 2001; Godoy-Diana et al. 2004), but the structure form is maintained. Their initial values $L_{h 0}$ and $L_{v 0}$ together with the initial translation velocity $U_{0}$ are used to calculate the control parameters $F_{h 0}, R e_{0}$ and $\alpha_{0}$ defined in the previous section.

The basic internal wave field in the present experiments follows the well known St Andrews cross pattern emitted by an oscillating cylinder. The control parameters 
(a)

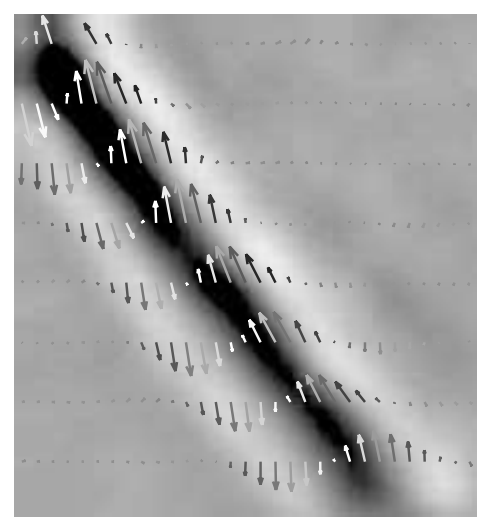

(b)

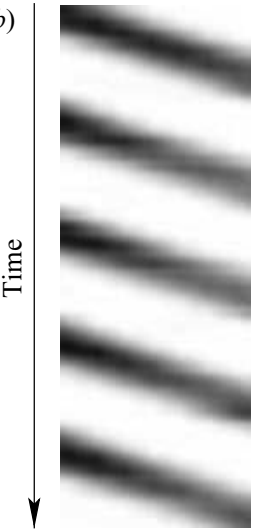

(d)
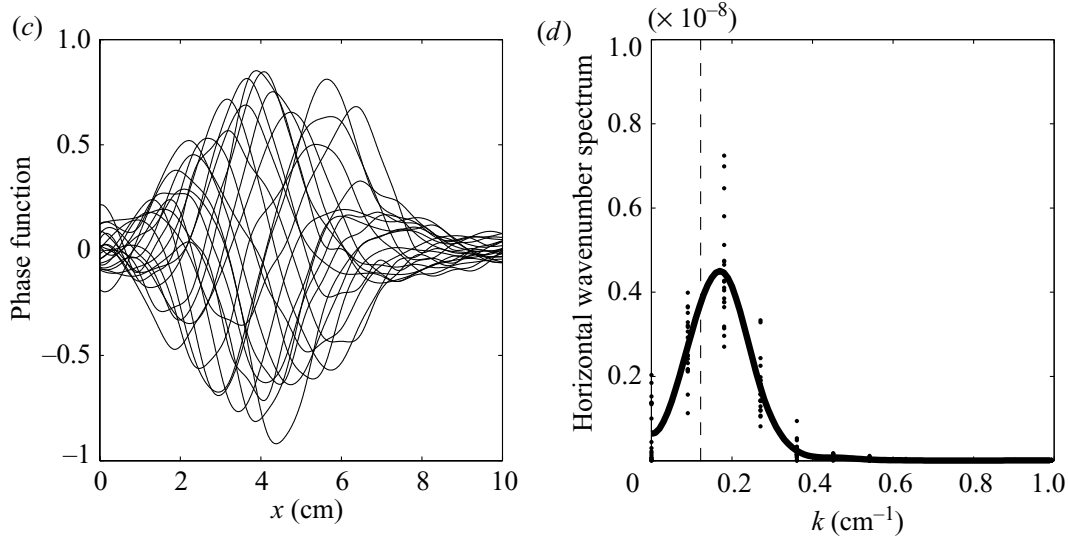

FiguRE 6. (a) PIV measurements on a vertical plane for one of the wave beams produced by an oscillating cylinder. Sets of vectors are shown with horizontal vorticity as background image. (b) Spatio-temporal diagram of a single horizontal line of $(a)$ plotted in the abscissa versus time in the ordinates. The horizontal phase velocity is the slope of the dark and light stripes. (c) Plots of the phase function sampled on a horizontal line at a fixed height and different times throughout the wave cycle permit us to visualize the wave packet. $(d)$ Dots and their mean value plotted in the solid line represent the spatial frequency content of the phase functions in $(c)$. The vertical dashed line marks the value of $k_{x}=\omega / c_{x}$ with $c_{x}$ obtained from the slope of the fringes in the spatio-temporal diagram $(b)$.

for each wave beam (figure $6 a$ ) are the cylinder oscillation frequency $\omega$ and the wavenumber. In practice, the wavenumber can be characterized by the horizontal wavenumber and measured in different manners: a first calculation can be obtained using the horizontal projection of the phase velocity as $k_{x}=\omega / c_{x}$. The horizontal phase velocity $c_{x}$ can be measured as the slope of the stripes that appear in a spatio-temporal diagram (figure 6b). This calculation gives the most accurate measure of the dominant wavenumber, however, since the beam is spatially localized and narrow, it cannot be appropriately represented by a single wavenumber. The continuous wavenumber spectrum contained in a wave beam can be estimated using Fourier transforms in space of a time series of measurements on a horizontal line (figure $6 c, d$ ). The vertical dashed line in figure $6(d)$ corresponds to the value of $k_{x}$ calculated with the phase velocity from the spatio-temporal diagram.

Various wave fields were used to observe different configurations of wave-vortex interaction. As hinted in the previous section, the basic design parameter was the 

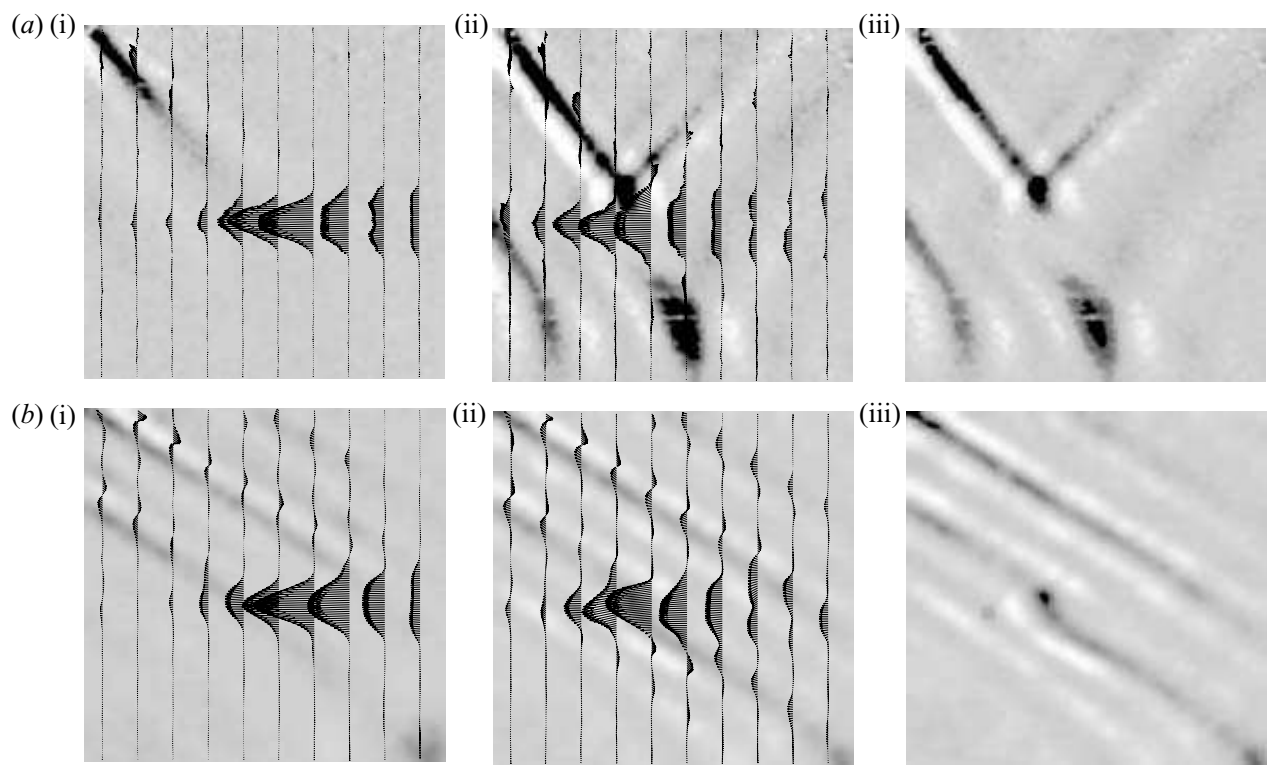

FIGURE 7. Velocity vectors and vertical velocity field obtained from PIV measurements on the vertical symmetry plane of the dipole for the counterpropagating configuration. The cylinder generating the waves is out of the viewing field at the top left-hand corner and the oscillation has been started at $t=30 \mathrm{~s}$ after the dipole was formed. The wave frequency is $(a) \omega=0.2 \mathrm{~s}^{-1}$ and $(b) \omega=0.13 \mathrm{~s}^{-1}$. In both cases (i) was taken at $t=80 \mathrm{~s}$ and (ii) at $t=115 \mathrm{~s}$. (iii) is the same as (ii), but only the vertical velocity field is drawn. Each frame shows an area in the $(x, z)$-plane of $38 \times 36 \mathrm{~cm}^{2}$. The same grey scale is used for the three frames of each case so that black and white represent $\pm 0.003 \mathrm{~m} \mathrm{~s}^{-1}$ in $(a)$ and $\pm 0.0012 \mathrm{~m} \mathrm{~s}^{-1}$ in $(b)$.

choice of the direction of the horizontal wave vector of interest with respect to the translation velocity of the dipole, in practice, determined by the position of the oscillating cylinder with respect to the screen that cuts the dipole. The control parameter was then the wave frequency, which was varied up to values allowing us to see either a critical layer in the copropagating case or a turning point in the counterpropagating case.

\section{Interactions}

\subsection{Waves in the dipole field}

In figure 7, we examine measurements on the vertical symmetry plane of the dipole for two cases with the set-up described in figure $3(c)$, that is, with waves counterpropagating with respect to the dipole. All parameters are the same except for the internal wave frequencies of figure $7(a) \omega=0.2 \mathrm{~s}^{-1}$ and figure $7(b) \omega=0.13 \mathrm{~s}^{-1}$ which determine the different angles of propagation with respect to the horizontal. The initial dipole parameters are $F_{h 0}=0.06, R e_{0}=131$ and $\alpha_{0}=0.4$. In both cases, the vertical velocity $u_{z}$ field and the velocity vectors are shown for times $t=80 \mathrm{~s}$ (before the interaction) and $t=115 \mathrm{~s}$ (after the waves have encountered the dipole). The second time is reproduced in a third frame without the vectors to allow for a clearer picture of the $u_{z}$ field. The vertical velocity field $u_{z}$ is particularly appropriate for revealing the internal wave pattern because, even though the velocity associated with the dipole is approximately five times larger than the velocity associated with the wave, the low-Froude-number dynamics determine that the vertical component 


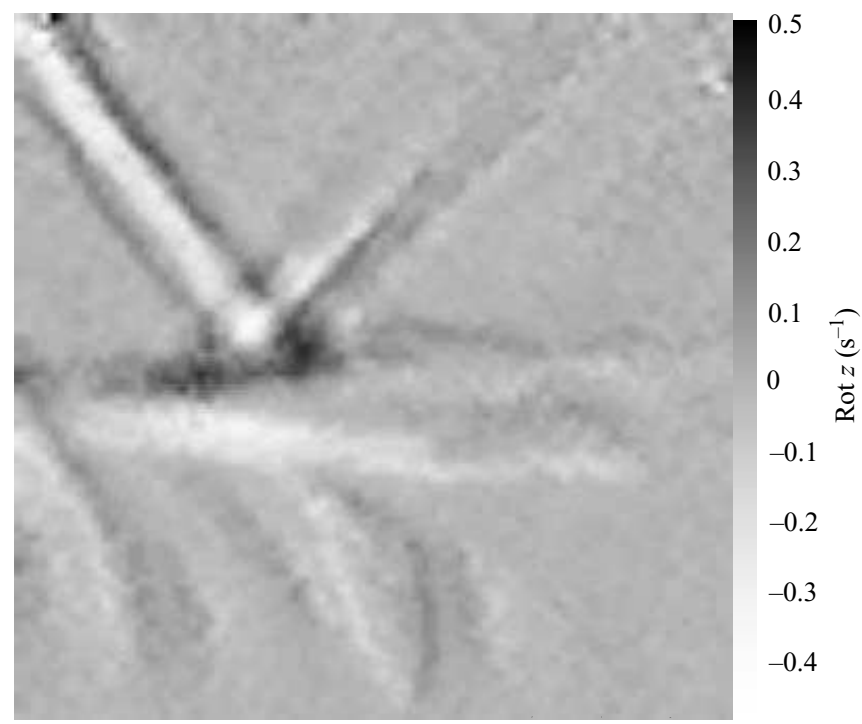

FIGURE 8. Horizontal vorticity field for $t=115 \mathrm{~s}$ and the same view as figure 7 .

of velocity is more than an order of magnitude smaller in the dipole than in the wave. In figure $7(a)$ at time $t=80 \mathrm{~s}$, the wave field emitted by the cylinder farthest to the right-hand side can be seen on the top-left-hand corner (we will refer to it hereinafter as the first beam) while the vector field reveals the core of the dipole at the centre of the image. At time $t=115 \mathrm{~s}$, the first beam has encountered the dipole core and, remarkably, a reflection of the wave is observed. The wave beam produced by the middle cylinder (the second beam) is also visible on the bottom left-hand side of the frame and it is barely starting to be affected by the dipole. The absence of waves from the first beam in the layers where the dipole core is passing indicates an episode of total reflection. (We recall that we are looking at the wave field in the vertical symmetry plane so that the reflection episode is total only in this midplane. As will be discussed in the following, the wave beam in other vertical planes may not be reflected.) The wave train reappears below the dipole, but it is distorted with respect to the original wave. It exhibits a wider beam and a larger amplitude when compared to the waves of the second beam to its left which did not interact with the dipole field. (A slight bending of both beams can be observed near the bottom of the image. This is due to a distortion of the linear background stratification caused by the proximity of the tank floor.) The reflected wave is best seen on the horizontal vorticity field (figure 8) where it can be seen that the reflected beam width (i.e. the dominant wavelength) is smaller than that of the direct beam.

A radically different situation is observed in the case with a lower frequency (figure $7 b$ ). The waves are not reflected, but a strong bending of the beam towards the vertical results when it passes through the dipole field. The beam under the dipole recovers the same direction as it had before interacting with the dipole field, but it should be noted that the wave amplitude is intensified (as shown by the higher contrast between the dark and light parts of the beam in the dipole and below it than those over it).

We now examine the case of waves where the horizontal component of the wave vector is in the same direction as the dipole velocity field, i.e. the copropagating configuration of figures $3(b)$ and $3(d)$. In figure 9 , the sequence presents both the 

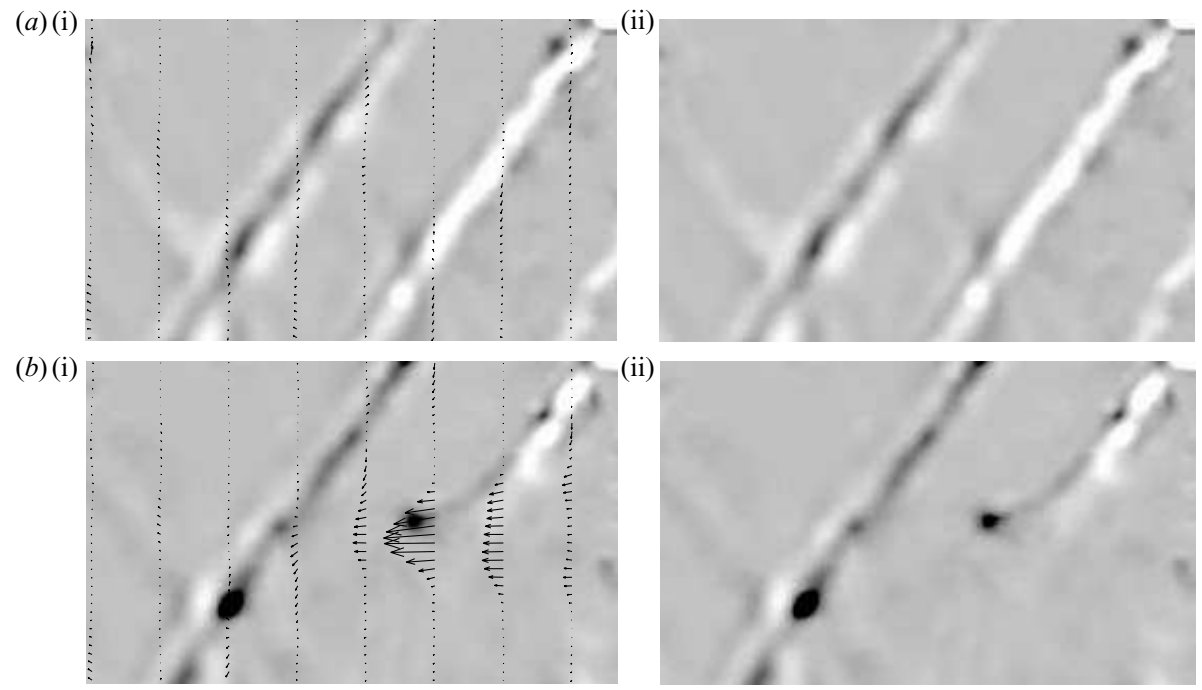

(ii)
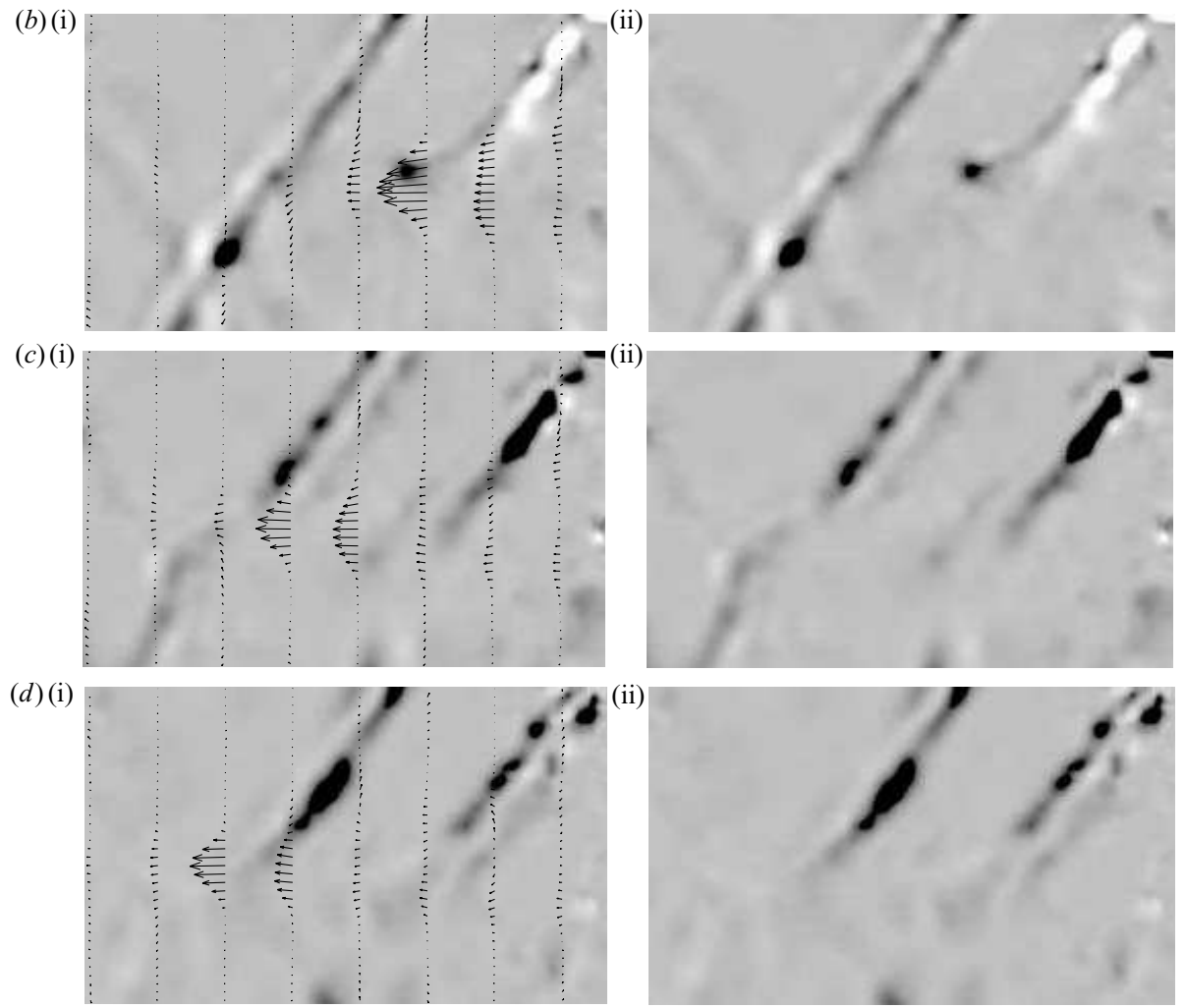

(ii)

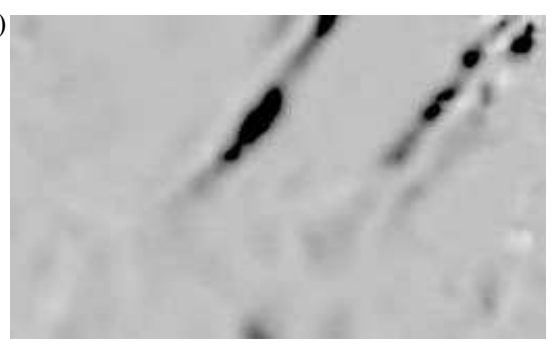

Figure 9. Vertical velocity field of internal waves of frequency $\omega=0.2 \mathrm{~s}^{-1}$ in the copropagating configuration (i.e. with the horizontal component of the wave vector in the same direction as the dipole velocity field) on the vertical symmetry plane for times $6,34,45$ and $53 \mathrm{~s}$, from top to bottom. Vertical sets of velocity vectors on the left-hand images show the position of the dipole, which moves from right to left. The oscillating cylinder is out of view near the top right-hand corner and the most visible wave beams are the one coming directly from the cylinder and another one that is reflected at the surface. Each frame shows an area in the $(x, z)$-plane of $44 \times 29 \mathrm{~cm}^{2}$. The same grey scale is used for all frames and the vertical velocity range is $\pm 0.003 \mathrm{~m} \mathrm{~s}^{-1}$.

velocity vector field as arrows and the vertical velocity in grey scale on the vertical symmetry plane of the dipole. The initial control parameters were $F_{h 0}=0.18, R e_{0}=182$ and $\alpha_{0}=1.27$ which describe a taller and faster dipole than the case described above. The experimental protocol was also different since the wave field was first established to give time for the reflected waves to reach the test section. This has the disadvantage of producing residual wavy motions in the background owing to the multiple reflections, which results in a noisier measurement. The wave field prior to 
the launching of the dipole is shown in figure $9(a)$. The frequency of the waves in this case was $\omega=0.2 \mathrm{~s}^{-1}$. The main beams in the field of view are the direct beam emanating from the upper right-hand corner of the image (the cylinder was placed out of view of the frames shown), and the reflection on the surface of the beam going up and to the left of the cylinder. In figure 9(a)(i), the former appears as a mainly white slanted stripe, whereas the latter is a parallel stripe mainly dark because of the quadrature phase shift due to reflection. Black and white in the grey scale represent, respectively, negative and positive vertical velocities associated to the wave motion. The evolution of the dipole can be monitored throughout the sequence by the sets of velocity vectors drawn over each image. We may point out that the effect of the vertically sheared motion produced by the dipole induces first a deformation of each wave beam, which are slightly bent following the advective background motion, and subsequently it 'erases' the beam in and, more remarkably, under the dipole (see for instance the reflected beam in figure $9(c)(\mathrm{i}))$.

\subsection{Two-dimensional rays}

The experimental observations of figures 7 and 9 show the deformation of internal wave beams that propagate through a background flow and motivate the interpretation in terms of ray theory. Particularly interesting is the confrontation of a theoretical prediction for the critical levels with the experimental observations of the critical behaviours. We start from the simplest approach that consists in looking at the vertical symmetry plane of the dipole, forgetting its three-dimensional structure. The application of equations (2.8) and (2.9) is straightforward using the experimental values for $\omega, k_{x}, U$ and $N$. The location of critical levels for a wave of frequency $\omega$ and horizontal wavenumber $k_{x}$ can be calculated for each background velocity $U$ field using the relative frequency $\omega_{r}$ that is a function of space and time:

$$
\omega_{r}=\omega-k_{x}|U|,
$$

where $\omega_{r}=0$ identifies the theoretical prediction for the location of a critical layer while $\omega_{r}=N$ corresponds to that of a turning point. Given the time-dependence of the background velocity field, it should be noted that we will calculate snapshots of the $\omega_{r}$ fields. In figure 10, we show contour levels of a $\omega_{r}-N$ field that corresponds to the counterpropagating case of figure 7 . The vertical velocity fields corresponding to figures $7(a)$ and $7(b)$ are reproduced in figures $10(a)$ and $10(c)$ while the respective $\omega_{r}-N$ fields are shown in figures $10(b)$ and $10(d)$. These fields were computed using the horizontal velocity $U(x, z)$ measured in an experiment of a dipole with no waves. The horizontal wavenumber in (5.1) was calculated as $k_{x}=\omega / c_{x}$, using the phase velocity $c_{x}$ measured on spatio-temporal diagrams as shown in figure 6 (this corresponds to the dashed vertical line in figure $6 d$ ). Regions where $\omega_{r}>N$ exist only for the first case, and the contour where $\omega_{r}=N$ is traced in figures $10(a)$ and $10(b)$ as a solid line. A critical band instead of a single contour can be estimated if we take into account that the wave packet is not monochromatic but corresponds to a wavenumber interval that can be defined from the spectral content of the wave packet. The two dashed lines plotted on figure $10(a)$ correspond to the limits of this band, calculated using the $k_{x}$ interval $\left(k_{x M I N}, k_{x M A X}\right)$ defined through figure $6(d)$ as the values where the spectrum mean curve is half its maximum value. The agreement between the observation and the theoretical prediction for the turning point is satisfactory, and this is quite surprising because the hypothesis of slow variation in space and time of the background flow at the base of ray theory is not actually respected, the velocity field of the dipole varying vertically as fast as the wave field. The space and 

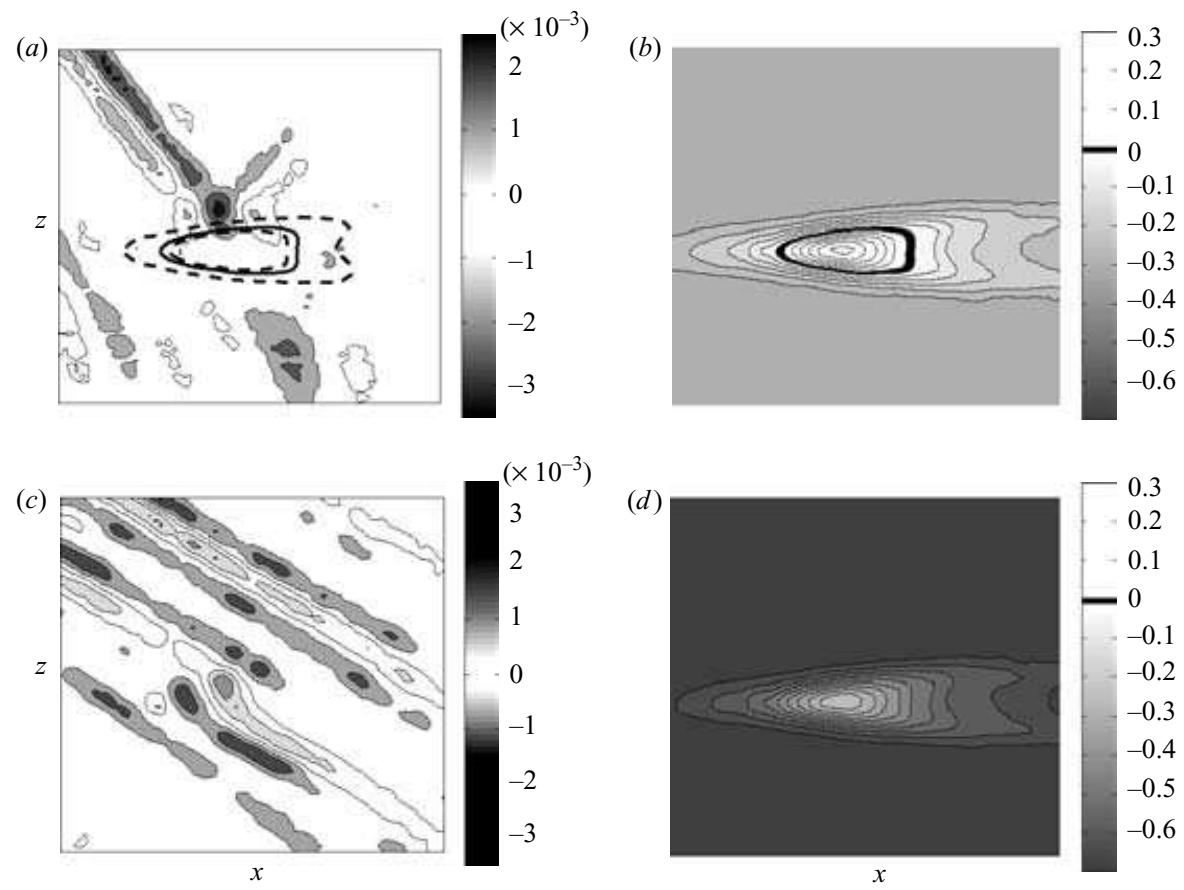

FIGURE 10. $(a, c)$ Vertical velocity and $(b, d) \omega_{r}-N$ fields corresponding to the $t=115 \mathrm{~s}$ frames of figure 7. $(a)$ and $(b)$ show the case of figure $7(a)$ where $\omega=0.2 \mathrm{~s}^{-1}$ and $(c)$ and $(d)$ that of figure 7(b) where $\omega=0.13 \mathrm{~s}^{-1}$. The location of the $\omega_{r}=N$ contour is drawn in solid line in $(a)$. Dashed lines show the limits of the critical band (see text.)

time dependence of these contours results from the evolution of $U(x, z ; t)$ so that, as the dipole translates, the contours move and eventually the critical reflection of one particular ray stops.

Figure 11 is the equivalent of figure 10 but for the copropagating configuration of figure 9. The critical contours $\omega_{r}=0$ are traced in figures 11(a), 11(c) and 11(e) on top of the vertical velocity fields that correspond, respectively, to the observations in figures $9(b), 9(c)$ and $9(d)$. For clarity, only the boundaries of the theoretical critical band calculated with the horizontal wavenumber spectrum limits are drawn in solid lines. While in figure 11(a) both limit calculations lead to a critical contour, in figure 11(c) the background velocity of the dipole has diminished enough to erase the inner contour and in figure 11( $e$ ) the critical band has entirely disappeared. The corresponding $\omega_{r}(x, z)$ fields are plotted in figures $11(b), 11(d)$ and $11(f)$. As in the previous case, these fields were calculated using $U(x, z)$ data of a control experiment without waves. The location of the theoretical critical layer in figure 11(a) coincides with the maximum deformation of the wave beam evidenced by the vertical velocity field. In contrast with the case of the turning point where the wave is totally reflected, in a critical layer all the wave energy is transferred to the background flow.

Going further on the two-dimensional interpretation, we could expect the waves that reach a critical layer to transfer momentum to the dipole and consequently retard its decay. An estimate of the transfer can be made using a simple momentum balance where all the wave momentum flux is thought to be transferred to the dipole at the critical level. The change in kinetic energy $E_{d}$ per unit mass of the dipole owing to the interaction in a control volume of dimensions given by the horizontal $\left(L_{h}\right)$ and 

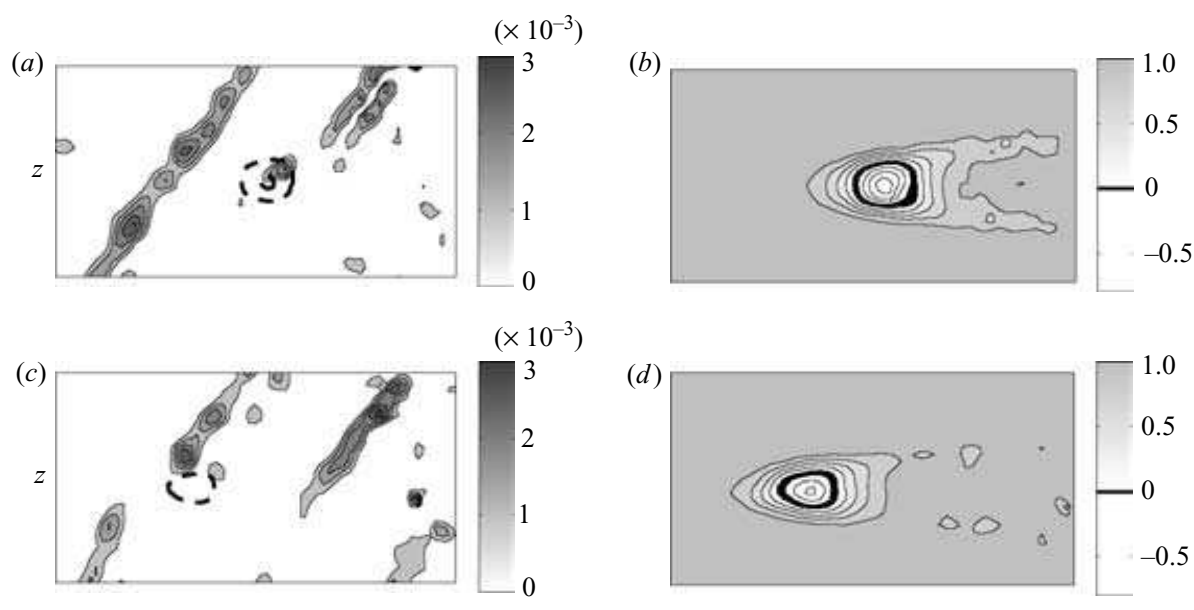

$\left(\times 10^{-3}\right)$
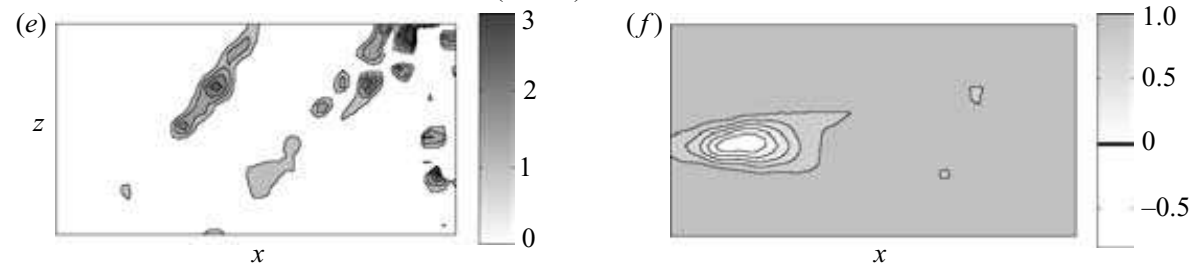

FiguRE 11. $(a),(c)$ and $(e)$ vertical velocity and $(b),(d)$, and $(f) \omega_{r}(x, z)$ fields corresponding to figure 9. The limits of the $\omega_{r}(x, z)=0$ critical band (see text) are drawn by a dashed line in $(a),(c)$ and $(e)$. Only the outer bound appears in $(c)$ and no theoretical critical band exists in $(e)$.

vertical $\left(L_{v}\right)$ length scales of the dipole can be written as

$$
\pi L_{h}^{2} L_{v} \frac{\mathrm{d} E_{d}}{\mathrm{~d} t}=S I
$$

where $S$ is the horizontal surface through which the wave energy flux on the vertical per unit mass $I$ enters the control volume. From equation (2.2), the wave energy flux can be written as $I=\langle E\rangle c_{g z}$ where the wave energy per unit mass averaged over a wave period $\langle E\rangle_{w}=\left\langle|\boldsymbol{u}|^{2}\right\rangle_{w}$ is obtained from the two-dimensional measurements on a vertical plane and the magnitude of the vertical component of the group velocity $c_{g z}$ computed from the measured wave frequency and wavenumber. The average kinetic energy per unit mass of the dipole during the time of the interaction is also obtained from the experimental data as $\langle E\rangle_{d}=\left\langle|\boldsymbol{u}|^{2}\right\rangle_{d}$ in the control volume. Two parameters to be estimated carefully are the surface $S$ and the time $\Delta T$ during which the transfer occurs. In the experiment, the actual transfer is not only limited by the 'spanwise' variation (i.e. in the $y$-direction, perpendicular to the two-dimensional measurements in the vertical midplane) of the dipolar field, which limits the extent of the critical condition, but also owing to the finite extent of the interaction region in the $x$ (or streamwise) direction. The latter determines, for instance, that the transfer may be limited because the waves leave the critical region before complete absorption. In the present order of magnitude calculation, we define $S=\lambda_{x}\left(L_{h} / 2\right)$, i.e. a rectangle of sides the horizontal 'wavelength' $\lambda_{x}$, equivalent to the beam width, and a fourth of the dipolar horizontal length scale $L_{h}$. The latter being the 'unit width' in which the 
(a)

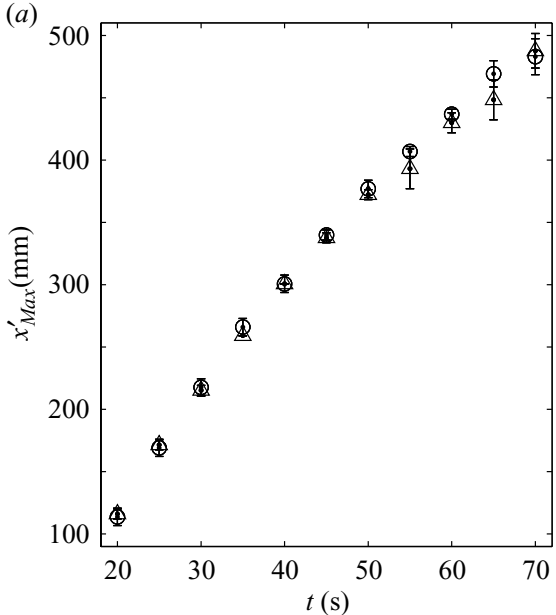

(b)

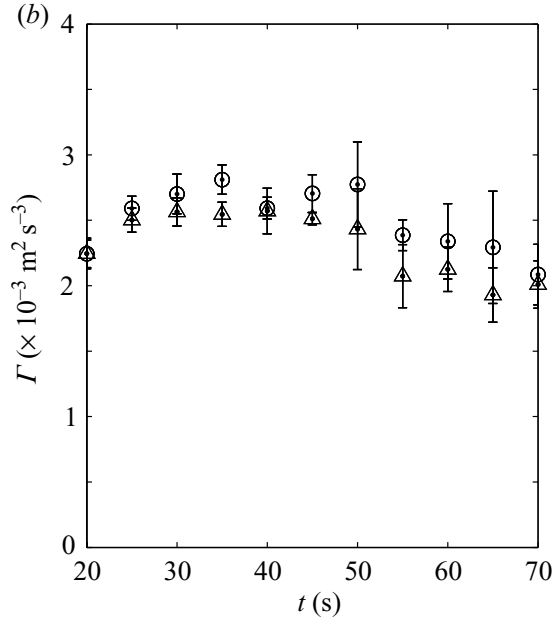

FIGURE 12. Evolution of the dipole for experiments with copropagating waves of frequency $\omega=0.2 \mathrm{~s}^{-1}(\bigcirc)$ and without any waves $(\triangle)$. $(a)$ Position of the maximum velocity $v s$. time and (b) circulation of a dipole half $v s$. time. Each point is the mean value of four experiments and the error bars represent the standard deviation. The experiments with waves correspond to the case shown in figures 9 and 11 .

two-dimensional critical condition is assumed to be approximately valid. Additionally, the time of interaction $\Delta T=L_{h} / U_{d}$, where $U_{d}$ is the mean translation velocity of the dipole, was estimated using the lapse during which the position of the beam overlaps with the critical region described by the outer dashed line in figure 9. The order of magnitude of the dipolar flow energy increase owing to the absorption of wave energy is thus estimated, upon substitution of the previous assumptions in equation (5.2), as

$$
\Delta E_{d} \sim \frac{\lambda_{x} c_{g}}{2 \pi L_{v} U_{d}}\left\langle|\boldsymbol{u}|^{2}\right\rangle_{w} .
$$

Computing this prediction for the experimental data corresponding to figure 9 and comparing it with the mean kinetic energy of the dipole during the interaction $\langle E\rangle_{d}=\left\langle|\boldsymbol{u}|^{2}\right\rangle_{d}$ gives a $\Delta E_{d} /\langle E\rangle_{d}$ ratio of $1 / 20$. This corresponds to a small value of the predicted change in the dipolar translation velocity due to the waves. Although the wave-vortex interaction is thus expected to be weak, it should nonetheless be observable through the analysis of the dipolar field evolution.

\subsection{Dipole evolution in presence of waves}

To measure the effect of the waves on the dipole, we compare the evolution of the dipole passing through the internal wave field with that of a freely evolving dipole (i.e. with no waves). In figure 12, we look at the evolution of the dipole of the experimental configuration in figure 11 through plots of $(a)$ dipole displacement (horizontal position of the horizontal velocity maximum measured on the dipole axis, defined by the crossing of the vertical and horizontal symmetry planes, versus time) and (b) dipole circulation versus time (defined from the horizontal velocity field integrated on the dipole axis). Experiments with copropagating waves $(\bigcirc)$ and without any waves $(\triangle)$ are plotted together. The displacement of the dipole is almost identical for the experiments with and without waves, a fact coherent with the maximum energy transfer estimated above, the limitation being the difficulty in generating intense and homogeneous wave fields in the experiment. In order to make the difference significant compared to the 
variability of the measurements, each experiment was repeated four times. (To avoid biasing due to eventual residual motion in the experimental tank, a $4 \mathrm{~h}$ rest time has been respected between two experiments. Also, to minimize any dissipating effect (due eventually to diffusion of salt or heat, or any other uncontrolled small forcing such as evaporation or wind stresses at the surface) experiments alternately with and without waves have been carried out, taking care also to alternate the time of the day when the experiments were performed.) The standard deviation of each measurement on figure 12, reported as error bars, are remarkably small, demonstrating the high degree of repeatability of our experimental procedure to create a pancake dipole. With such a sharp measurement, the systematically faster propagation of the dipole in the presence of the wave is significant in spite of its being very weak. The maximum displacement difference between the propagation with and without waves is at maximum equal to a standard deviation of each single measurement. The average of four independent events has a standard deviation approximately equal to half the standard deviation of a single realization. In the analysis of figure 12, this means that from time $t=55 \mathrm{~s}$ to $t=70 \mathrm{~s}$ the mean displacement of the dipole in presence of waves is significantly larger than its counterpart without waves. The difference is even more significant when considering the dipole circulation on the axis that is higher by 10 to $20 \%$ in the presence of gravity waves compared to the reference case without waves. The equivalent analysis for the counterpropagating experimental configuration of figure 10 , i.e. for the case where the turning point and reflection of the wave were observed, is not presented here because it gives no measurable modification of the dipole displacement and vortex circulation, as expected from two-dimensional theory. This is confirmed by the plots in figures $13(a)$ and $13(b)$.

In figure 13, we compare contour plots of the horizontal velocity on a vertical plane and vertical profiles passing through the point of maximum velocity in cases without and with gravity waves and in both counterpropagating and copropagating configurations. In figure $13(a)$, the dipole without any wave field is traced as a solid line. The dashed line shows the dipole with the counterpropagating wave of frequency $\omega=0.2 \mathrm{~s}^{-1}$ corresponding to figure $7(a)$ for $t=115 \mathrm{~s}$. The dashed contours are perturbed by the presence of the waves. Figure 13(b) shows the corresponding vertical profile at the dipole centre of the horizontal velocity. Almost no difference can be seen in the profiles between the cases without and with counterpropagating waves. This confirms that when reflection takes place it imposes no modification on the vortex motion. The same comparison between the horizontal velocity field (and respective profiles) of the dipole without and with copropagating waves is presented in figures $13(c)$ and $13(d)$. Strikingly, the velocity profile shows a systematic larger velocity on the upper part of the dipole that corresponds to the region where the wave, coming from above, is absorbed in a critical layer. Although modest (less than $10 \%$ ), this increase is significant, in particular, when compared to the lower part of the dipole not reached by the wave, which is strictly identical to the reference velocity profile in the absence of waves.

\subsection{Horizontal plane measurements: three-dimensional ray refraction}

Up to now we have presented a two-dimensional view of the wave-vortex interaction based on measurements on the vertical symmetry plane of the dipole. In this section, we examine the three-dimensional structure of the dipolar field and the wave beams using PIV measurements on horizontal planes. In figure 14, measurements on the horizontal midplane of the dipole are shown for both $(a)$ the counterpropagating and (b) the copropagating configurations during the interaction. Both cases correspond 

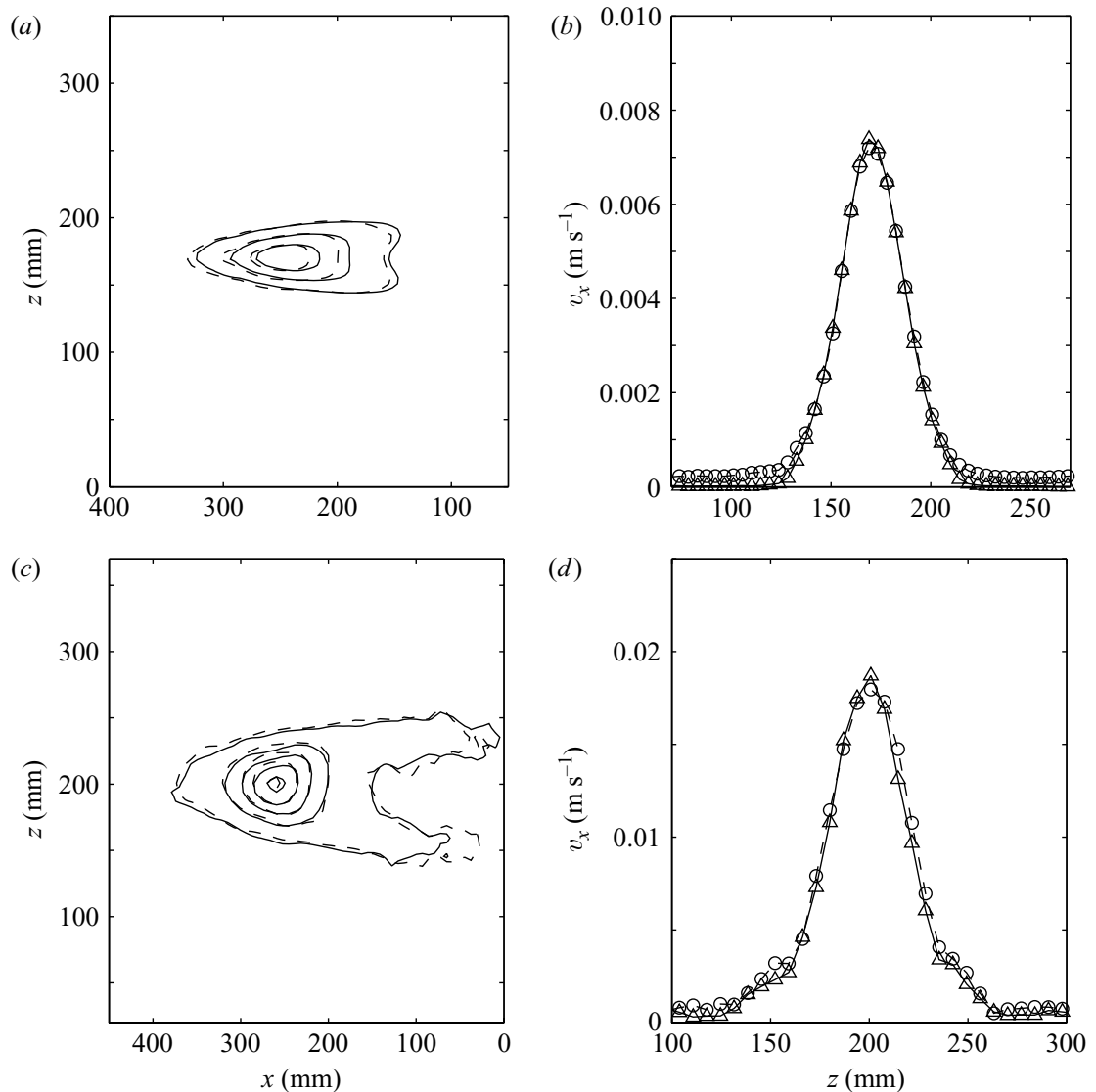

FIGURE 13. Contour plots and vertical profiles of the horizontal velocity field for $(a)$ and $(b)$ the dipole of figure 7 and $(c)$ and $(d)$ the dipole of figure 9. Dashed lines and $\bigcirc$ symbols correspond to experimental configurations with waves of frequency $\omega=0.2 \mathrm{~s}^{-1}$. Solid lines and $\triangle$ symbols are the control case without waves. Note that a different aspect ratio has been used in the copropagating and contrapropagating cases explaining the difference between $(a)$ and $(c)$.

to parameter values for which no critical conditions are verified so that the wave beam traverses the dipole layers downwards. In each case, (i) and (ii) show velocity vectors traced, respectively, over fields of the vertical vorticity $\left(\partial u_{x} / \partial y-\partial u_{y} / \partial x\right)$ and the divergence of the horizontal velocity field $\left(\partial u_{x} / \partial x+\partial u_{y} / \partial y\right)$. While the velocity vectors let us visualize the position of the dipole, the waves cannot be distinguished owing to the smallness of the velocities associated to the waves compared to those associated to the dipole. The waves are only visible looking at the divergence field where the lines of constant phase appear as dark and light stripes. (The wave/vortex decomposition of the flow in a non-divergent vortex mode containing vertical vorticity and a wave mode that contains all the vertical velocity (as discussed e.g. by Staquet \& Riley 1989; Riley \& Lelong 2000) is found to be very useful in the analysis of the flow in a horizontal plane because, while the dipole can be observed through the vorticity field (that for the Froude numbers used here is, in practice, identical to the potential vorticty), the vertical velocity associated to wave motion induces by conservation of mass a non-zero divergence of the horizontal flow that, although small, is measurable in the experiment.) The divergence fields are noisy because 
(a) (i)

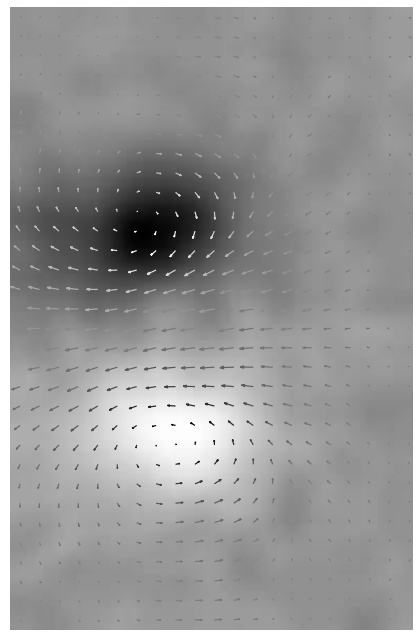

(b) (i)

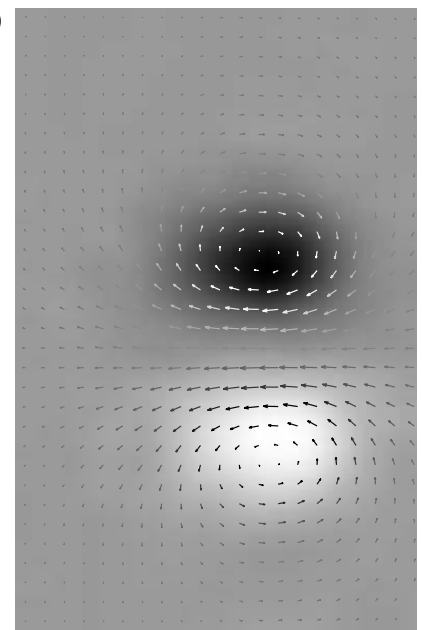

(ii)

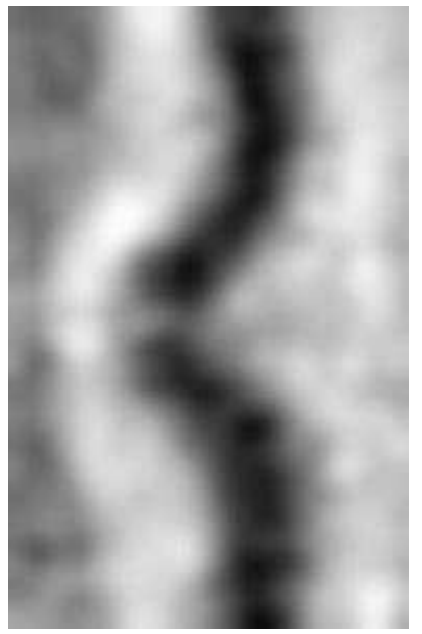

(ii)

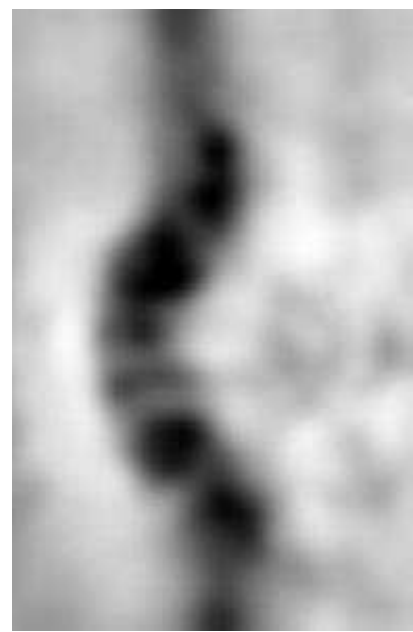

FIGURE 14. PIV measurements on the horizontal midplane for waves interacting with a dipole in the $(a)$ counterpropagating and $(b)$ copropagating configurations. The wave frequencies are (a) $\omega=0.11 \mathrm{~s}^{-1}$ and (b) $\omega=0.13 \mathrm{~s}^{-1}$. (i) Vertical vorticity; (ii) divergence of the horizontal velocity field. Velocity vectors measured are shown in all frames. Field of view: $16.5 \times 25.5 \mathrm{~cm}^{2}$ in the $(x, y)$-plane. Grey scales: $(a) \pm 0.5 \mathrm{~s}^{-1}$ for vorticity and $\pm 0.06 \mathrm{~s}^{-1}$ for divergence; (b) $\pm 0.6 \mathrm{~s}^{-1}$ for vorticity and $\pm 0.05 \mathrm{~s}^{-1}$ for divergence.

they require the evaluation of derivatives that are dominated by the vortex motion. Therefore, the absolute noise level on the derivative owing to the PIV technique are about $10 \%$ of the maximum vorticity. The absolute error on the divergence is then also about $10 \%$ of the maximum vorticity. Since the wave field is weak, the associated maximum divergence is about $1 / 5$ of the vorticity and the relative noise on the divergence field is typically about $50 \%$. In order to obtain a clearer signal, the divergence fields shown in figures $14(a)$ (ii) and 14(b)(ii) are the average of three fields separated by half a wave-period (the divergence of the second field being multiplied by -1 in order to compensate its phase opposition with the two other fields). In both counterpropagating and copropagating cases, the effect of the dipolar field on the wave beam is readily identified as a deformation of the phase lines (which are straight 
(a) (i)

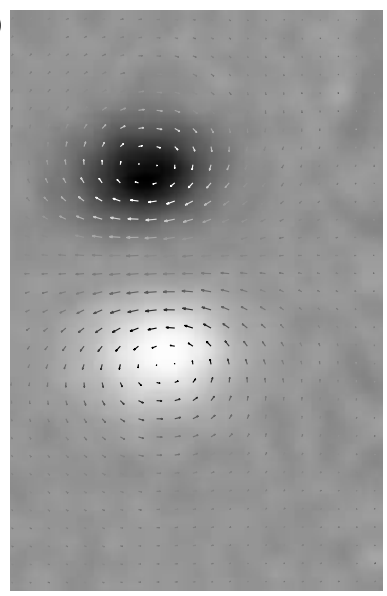

(b) (i)

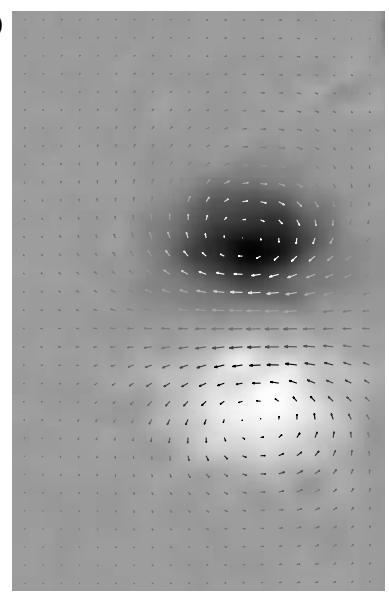

(ii)

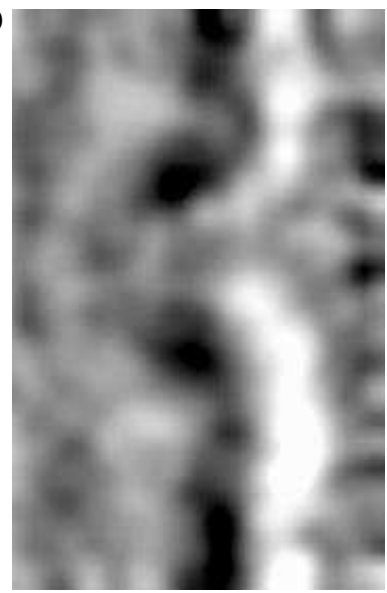

(ii)

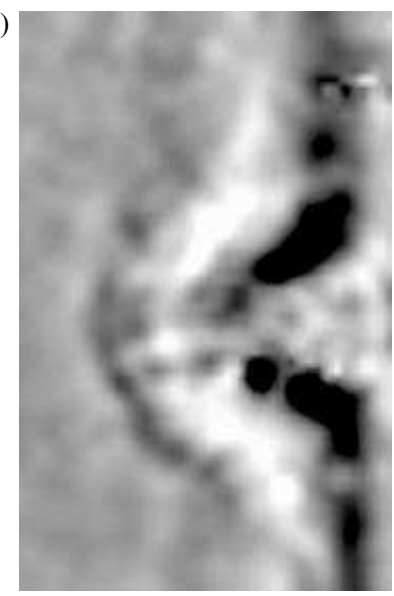

(iii)

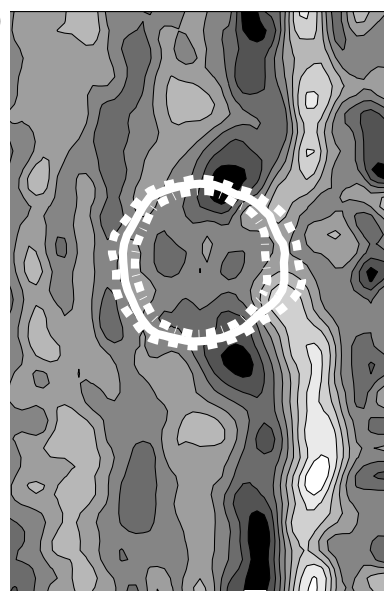

(iii)

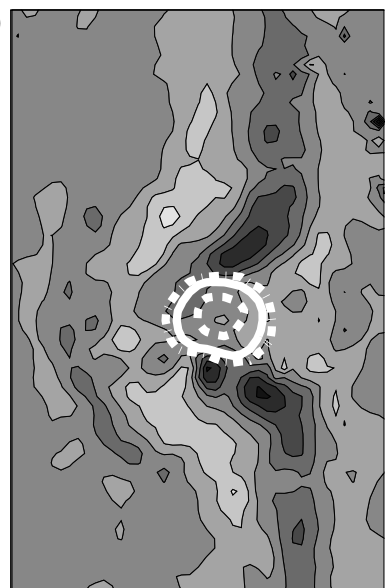

FIGURE 15. PIV measurements on the horizontal midplane for waves interacting with a dipole in the $(a)$ counterpropagating and $(b)$ copropagating configurations during a critical interaction. The wave frequency is $\omega=0.17 \mathrm{~s}^{-1}$ in both cases. (i) Vertical vorticity; (ii) divergence of the horizontal velocity field; (iii) critical contours of the $(a) \omega_{r}=N$ and $(b) \omega_{r}=0$ fields drawn over filled contour plots of the divergence field. The continuous contours represent the predictions using the phase velocity calculation and the dotted contours are the upper and lower bounds of the critical band (see also discussion of figures 10 and 11). In (i), (ii) the velocity vectors measured are shown. Fields of view: $16.5 \times 25.5 \mathrm{~cm}^{2}$ in the $(x, y)$-plane. Grey scales: (a) $\pm 0.8 \mathrm{~s}^{-1}$ for vorticity and $\pm 0.05 \mathrm{~s}^{-1}$ for divergence; $(b) \pm 0.75 \mathrm{~s}^{-1}$ for vorticity and $\pm 0.06 \mathrm{~s}^{-1}$ for divergence.

when there is no dipole). The phase lines marked by a dark stripe in figures 14(a)(ii) and $14(b)($ ii) are deformed as they are advected by the dipole in the central region that corresponds to the location of maximum velocity of the background dipolar velocity field. The wave beam deformed by the passing dipole nearly conserves its horizontal 'wavelength', defined as the horizontal beam width.

Figure 15 is equivalent to figure 14, but for cases corresponding to parameter values for which the critical conditions for $(a)$ a turning point and $(b)$ a critical layer are verified. A third frame is added in both cases $(a)$ and $(b)$ with the calculation of critical contours where $\omega_{r}=N$ and $\omega_{r}=0$, respectively (the horizontal counterpart of those shown in figures 10 and 11 for the vertical plane measurements). For the computation 


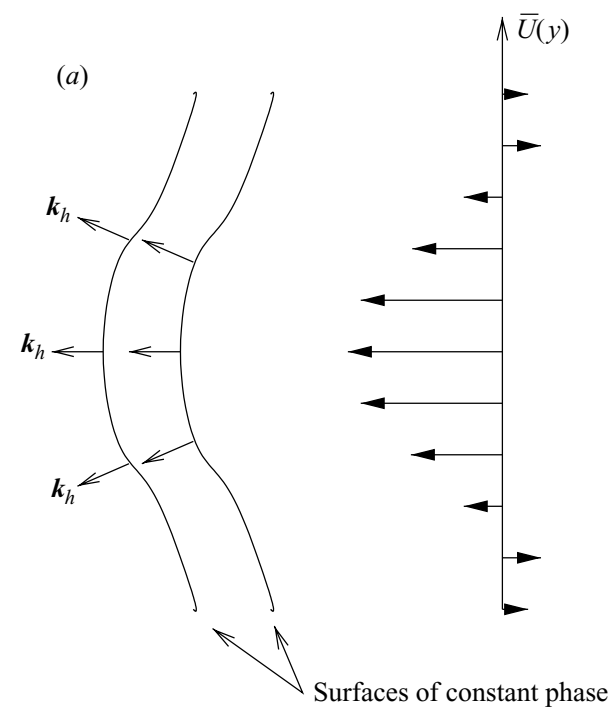

(b)

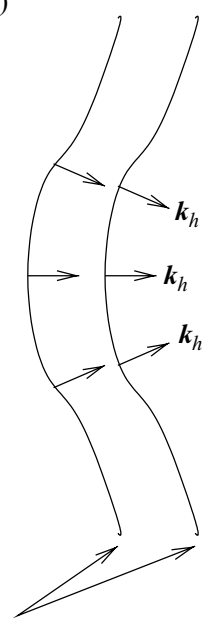

FigURE 16. Schematic diagram of the internal wave phase lines deformed by the dipolar field showing the refraction of the wave vector. In $(a)$ the critical-layer case where the horizontal wave vector $\boldsymbol{k}_{h}$ points in the direction of the mean dipolar field $U(y)$ (copropagating case) and the defocusing effect occurs owing to diverging horizontal wave vectors. The opposite picture is true for the turning point case $(b)$ where the isophases move opposite to the dipolar mean velocity (counterpropagating case) and wave focusing is produced as a result of converging $\boldsymbol{k}_{h}$.

of the relative velocity, the variations in the modulus and orientation of the horizontal wavevector have been neglected, as it should in the first-order approximation. In the counterpropagating case (figure 15(a)), the wave signal vanishes in the central region that corresponds to the maximum dipolar velocity. This is the 'forbidden zone' below the turning-point layer where the waves are not allowed because their relative frequency would be higher than $N$. It compares favourably with the critical contours $\omega_{r}(x, y)=N$ (figure $15 a$ (iii)). The copropagating case is similar, as shown in figure $15(b)$, where the wave almost vanishes in the forbidden region where $\omega_{r}<0$. The $\omega_{r}(x, y)=0$ contours give a quantitative measure of the horizontal extent of the critical wave-vortex interaction and were used as the characteristic 'spanwise' horizontal length scale for the energy transfer estimation made in equation (5.3).

The deformation of the phase lines demonstrated by the observations on the horizontal plane determines a three-dimensional effect not yet discussed: the refraction of the wave vector. We recall that for internal gravity waves, the horizontal projections of the phase velocity and the group velocity are parallel and therefore orthogonal to the horizontal phase lines. The observed bending of the phase lines, represented schematically in figure 16, implies that the horizontal wave vector field diverges in the case of copropagating waves, whereas it converges for counterpropagating waves. Wave energy propagating with the group velocity thus diverges or converges implying, respectively, a defocusing or focusing of the wave beam. The wave defocusing that accompanies the copropagating interaction explains the fact that the waves 'disappear' in the vertical plane view of figure 9 below the layers where the dipole passes even in the absence of a critical layer (figures $9(d)$ and 11e). The inverse effect is observed in the counterpropagating case where the wave beam is focused, as represented in figure $16(b)$. A confirmation of this focusing effect can be found in a further analysis of figures $7(a)$ and $7(b)$. In figure 7(a), the reappearance of waves below the dipole after 


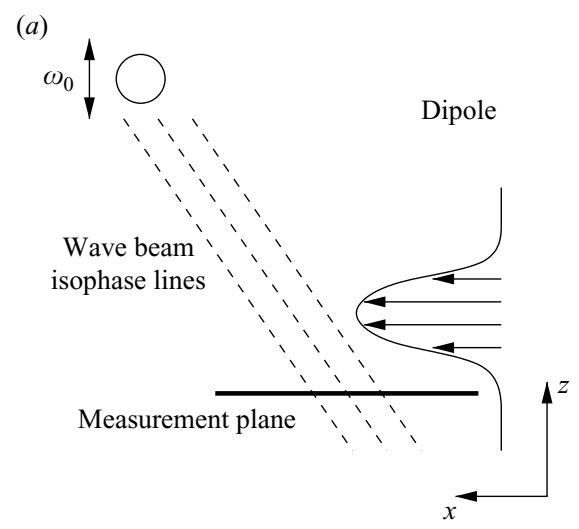

(b)
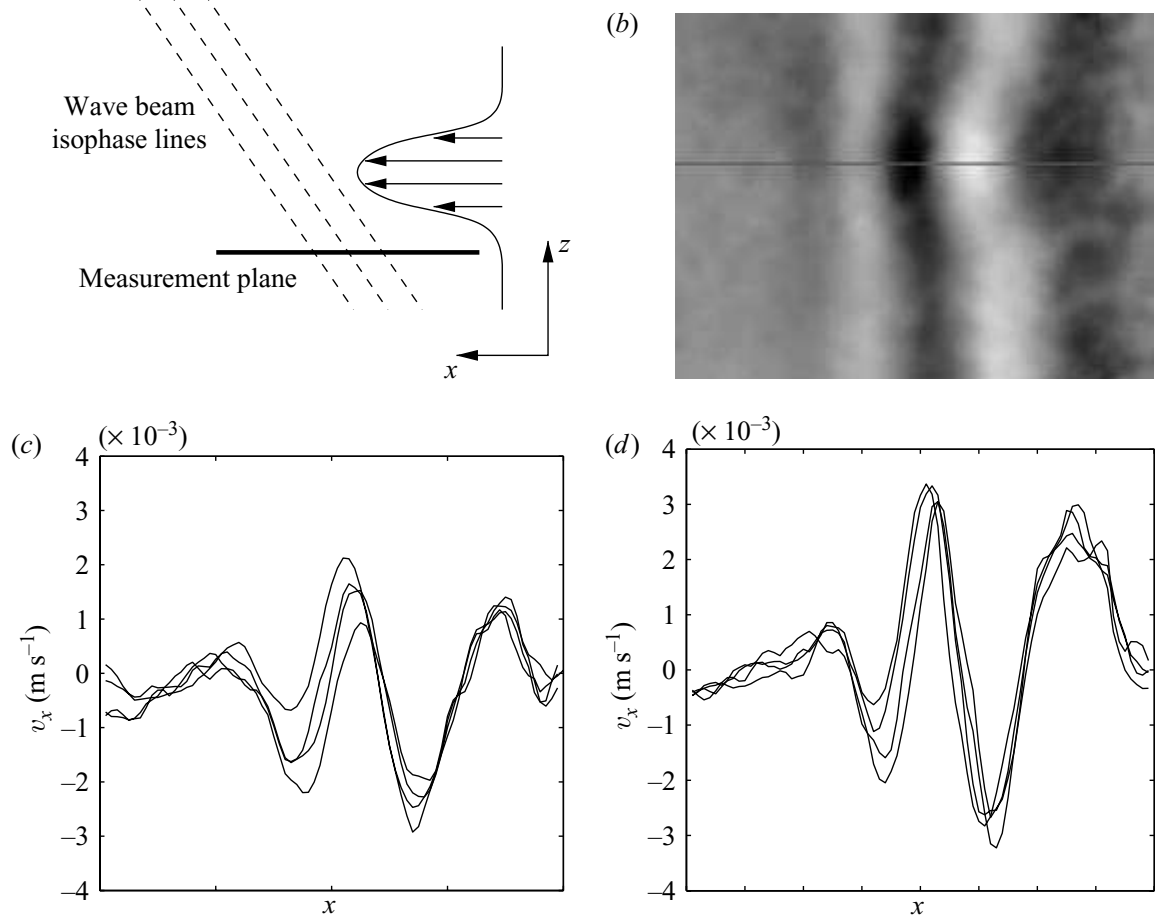

FIGURE 17. (a) Schematic view of the vertical plane showing the location of the horizontal measurements. $(b)$ Horizontal velocity field in the plane below the dipole. $(c, d)$ Profiles of horizontal velocity taken at the line shown in $(b)$ before and during the focusing event, respectively. The four profiles shown in each of plots $(c)$ and $(d)$ correspond to four snapshots separated by $1 / 16$ of the wave period. The field of view in $(b)$ is $31 \times 25.5 \mathrm{~cm}^{2}$ in the $(x, y)$-plane. The $x$-axis in $(c)$ and $(d)$ spans the whole horizontal dimension of $(b)$.

the episode of total reflection in the vertical midplane can be understood thinking of vertical planes parallel to this midplane where the measurements are made. In these off-centre planes, the maximum velocity is smaller than on the axis and, for a sufficient shift, the wave rays initially in these outer planes will not encounter the critical level and will pass through the dipole. However, because of the deformation of the isophase lines, the rays are no more in the off-axis plane after traversing the dipole, but focus towards the midplane, explaining the reappearance of waves below the dipole observed in figure 7(a). The same mechanism occurs in the case of figure $7(b)$. There, the wave frequency is not high enough for a reflection of the wave to be achieved, but the incoming beam is also deformed by the dipole and observation shows that the wave that traverses the dipolar field is intensified.

The issue of wave focusing by a dipolar field merits a further comment. An illuminating picture of this effect can be obtained using horizontal plane measurements below the dipole in the counterpropagating configuration (see figure 17). In figure 17(a), a schematic diagram of the vertical plane shows the location of the horizontal measurements shown in figure 17(b). The latter shows the $x$-component of the velocity field in the plane below the dipole during the focusing event. In this lower 
plane, the velocity due to the dipole is very small so that the horizontal velocity field can be used to visualize the waves, hence avoiding the use of the noisier divergence field. The wave amplitude is readily seen to be magnified in the region that crossed the core of the dipole. The experimental parameters used in this case are the same as in the case shown in figure 14(a) so that only the observation plane has been lowered. A comparison of velocity profiles taken at the horizontal line in figure 17(b) before (figure 17c) and during (figure 17d) the passing of the dipole gives evidence of intensification linked to the focusing event. The two sets of profiles were chosen at the same phase so that the different peaks of the wave-induced velocity can be compared directly. The focused wave amplitude is thus, in that particular plane, in average about $50 \%$ larger than the wave amplitude before the wave-vortex interaction.

\section{Conclusions}

The experimental study of wave-vortex interaction in a stratified fluid presented in this paper gives new evidence that three-dimensional effects may play a significant role in the occurrence of critical levels in wave propagation. Internal gravity waves are produced in the laboratory by oscillating either a single cylinder or a set of cylinders in a salt-stratified water tank. The waves interact with well-controlled free vortices produced by a gate of variable height placed in front of a double-flap mechanism Godoy-Diana et al. (2004). Different configurations of interaction permit us to observe waves that are shifted towards either a turning point, where the wave frequency $\omega_{r}$ in the frame of reference of the fluid tends to the buoyancy frequency $N$, or a critical layer, where $\omega_{r} \rightarrow 0$. Measurements of the velocity field on a vertical plane perpendicular to the wave surfaces of constant phase and aligned with the moving direction of the dipole give a picture of the deformation of the wave beams that encounter the dipolar field and allow us to identify waves that reach both singularities: a turning point and a critical layer. A two-dimensional analysis built on a classical ray-theory for internal gravity waves in a shear flow predicts the location of critical levels in good agreement with the observations (see figures 10 and 11 depending on the relative direction of the horizontal phase velocity of the wave component to the translation direction of the dipole). That the two-dimensional model succeeds in predicting the critical levels is remarkable, not only because, as discussed in $\S 5.2$, the shear flow produced by the dipole does not satisfy the hypotheses of slow variation underlying ray theory, but also because both the basic flow and the ray propagation should be considered three-dimensional.

Two questions arise in regard to the critical layer predicted by the two-dimensional model; On the one hand, what changes with respect to the two-dimensional interpretation should be expected as a result of the three-dimensional nature of the dipolar field? On the otherhand, can the wave energy transferred to the background flow at the critical layer induce an observable change on the dipole? The latter question was addressed using a simple energy balance in a control volume, assuming that all the wave energy on a beam that is bent towards a critical layer is transferred to the background flow (see §5.2) and a weak modification of the vortex flow was predicted owing to the limited amplitude of experimentally achievable internal gravity waves. By a rigorous and systematic experimental protocol, this momentum transfer was observed, the dipole evolution being monitored through its position and circulation versus time (figure 12) and through profiles of the horizontal velocity field in the vertical plane (figure 13). On the contrary, in the counterpropagating case, the same 
experimental procedure demonstrated the absence of retro effect on the vortices and so, even in cases of total reflection in the midplane.

Three-dimensional effects on the wave propagation were discussed exploiting measurements of the velocity field on horizontal planes (\$5.4). These revealed a deformation of the wave phase lines that are advected by the velocity field of the dipole, evidencing a phenomenon of wave refraction. The refraction of a wave beam leads to opposite effects when the wave and the dipole are copropagating or counterpropagating. In the copropagating case the horizontal wave vector field diverges, together with the horizontal group velocity vectors (figure 16a), determining the wave field to be defocused. This phenomenon determines as a first consequence that the horizontal extent of the critical-layer interaction is drastically limited. On the other hand, it might explain the disappearance of the wave beam below the dipole in figures $9(d)$ and $11(e)$ where the dipole velocity is not sufficient to bring the wave to a critical layer. In the counterpropagating case, the horizontal wave vector field converges (figure 16b). The expected focusing of the waves was confirmed firstly by re-examining the vertical plane observations for waves counterpropagating with respect to the dipolar velocity field (figure 7). In that case, a noticeable amplification of the wave amplitude in the part of the beam distorted by the dipolar field is observed. Also, even when total reflection occurs in the midplane, the focused wave reappears under the dipole. A second confirmation of the wave beam focusing by the dipolar field was obtained looking at PIV measurements on a horizontal plane below the dipole layers (figure 17). There, a $50 \%$ wave amplitude magnification occurs in the part of the wave that goes through the dipole core.

The ubiquitous presence in a geophysical context of internal gravity waves propagating through three-dimensional vortical flows renders the consideration of the three-dimensional effects studied here indispensable to an adequate modelling. Because of the strong limitation on the Reynolds number of low-Froude-number experiments, when critical layers occur in the laboratory they are of a viscous nature and, similarly, focusing points for waves are smoothed out by diffusion. This is unlikely to be the case in geophysical flows where Reynolds numbers are enormous. In that case, only nonlinearity, instability and turbulence associated to wave breaking may desingularize critical layers or focusing points. The focusing effects discussed here can radically change the conditions for wave breaking, for instance, the fact that a critical level need not be attained for a wave to break, and thus play an important role in the control of mixing and dissipation in atmospheric and oceanic flows.

We thank warmly Antoine Garcia for his invaluable help in the construction and installation of the wave-generating devices and Anne-Virginie Salsac for her careful reading of a first version of this paper. Support from CONACyT-México to R. G. D. through a scholarship for doctoral studies is gratefully acknowledged.

\section{REFERENCES}

AfanasYev, Y. 2003 Spontaneous emission of gravity waves by interacting vortex dipoles in a stratified fluid: laboratory experiments. Geophys. Astrophys. Fluid Dyn. 97, 79-95.

Bartello, P. 1995 Geostrophic adjustment and inverse cascades in rotating stratified turbulence. J. Atmos. Sci. 52, 4410-4428.

Beckers, M., Verzicco, R., Clercx, H. \& van Heijst, G. 2001 Dynamics of pancake-like vortices in a stratified fluid: experiments, model and numerical simulations. J. Fluid Mech. 433, 1-27.

Billant, P. \& Chomaz, J. 2000 Experimental evidence for a zigzag instability of a vertical columnar vortex pair in a strongly stratified fluid. J. Fluid Mech. 418, 167-188. 
Bonneton, P., Chomaz, J. \& Hopfinger, E. 1993 Internal waves produced by the turbulent wake of a sphere in a stratified fluid. J. Fluid Mech. 254, 23-40.

Bonnier, M., EifF, O. \& Bonneton, P. 2000 On the density structure of far-wake vortices in a stratified fluid. Dyn. Atmos. Oceans 31, 117-137.

Booker, J. \& Bretherton, F. 1967 The critical layer for internal gravity waves in a shear flow. J. Fluid Mech. 27, 513-539.

BRETHERTON, F. 1966 The propagation of groups of internal gravity waves in a shear flow. Q. J. R. Met. Soc. 92, 466-480.

Broutman, D., Macaskill, C., McIntyre, M. \& Rottman, J. 1997 On Doppler-spreading models of internal waves. Geophys. Res. Lett. 24, 2813-2816.

BüHLER, O. \& MCINTYRe, M. 1998 On non-dissipative wave-mean interactions in the atmosphere or oceans. J. Fluid Mech. 354, 301-343.

BüHLER, O. \& McInTYRe, M. 2003 Remote recoil: a new wave-mean interaction effect. J. Fluid Mech. 492, 207-230.

Edwards, N. \& Staquet, C. 2005 Focusing of an inertia-gravity wave packet by a baroclinic shear flow. Dyn. Atmos. Oceans 40, 91-113.

Fincham, A., Maxworthy, T. \& Spedding, G. 1996 Energy dissipation and vortex structure in freely decaying, stratified grid turbulence. Dyn. Atmos. Oceans 23, 155-169.

FlóR, J. \& van HeIJST, G. 1994 An experimental study of dipolar vortex structures in a stratified fluid. J. Fluid Mech. 279, 101-133.

FlóR, J. \& van HeIJst, G. 1996 Stable and unstable monopolar vortices in a stratified fluid. J. Fluid Mech. 311, 257-287.

Flór, J., van Heisst, G. \& Delfos, R. 1995 Decay of dipolar vortex structures in a stratified fluid. Phys. Fluids 7, 374-383.

Fritts, D. \& Alexander, M. 2003 Gravity wave dynamics and effects in the middle atmosphere. Rev. Geophys. 41, doi: 10.1029/2001RG000106.

Gill, A. 1982 Atmosphere-Ocean Dynamics. Academic.

Godeferd, F. \& CAmbon, C. 1994 Detailed investigation of energy transfers in homogeneous stratified turbulence. Phys. Fluids 6, 2084-2100.

Godoy-Diana, R. \& Chomaz, J. 2003 Effect of the Schmidt number on the diffusion of axisymmetric pancake vortices in a stratified fluid. Phys. Fluids 15, 1058-1064.

Godoy-Diana, R., Chomaz, J. \& Billant, P. 2004 Vertical length scale selection for pancake vortices in a strongly stratified fluid. J. Fluid Mech. 504, 229-238.

van Heisst, G. \& Flór, J. 1989 Dipole formation and collisions in a stratified fluid. Nature 340, 212-215.

JaVAm, A., Imberger, J. \& Armfield, S. 2000 Numerical study of internal wave-caustic and internal wave-shear interactions in a stratified fluid. J. Fluid Mech. 415, 89-116.

JAVAM, A. \& Redekopp, L. 1998 The transmission of spatially-compact internal wave packets through a critical level. Dyn. Atmos. Oceans 28, 127-138.

Koop, C. 1981 A preliminary investigation of the interaction of internal waves with a steady shearing motion. J. Fluid Mech. 113, 347-386.

LELONG, M. \& RiLEY, J. 1991 Internal wave-vortical mode interactions in strongly stratified flows. J. Fluid Mech. 232, 1-19.

Lelong, M. \& Sundermeyer, M. 2005 Geostrophic adjustment of an isolated diapycnal mixing event and its implications for small-scale lateral dispersion. J. Phys. Oceanogr. In press.

Lighthill, J. 1978 Waves in Fluids. Cambridge University Press.

Lighthill, J. 1996 Internal waves and related initial-value problems. Dyn. Atmos. Oceans 23, 3-17.

Lilly, D. 1983 Stratified turbulence and the mesoscale variability of the atmosphere. J. Atmos. Sci. 40, 749-761.

Lin, J. \& PaO, Y. 1979 Wakes in stratified fluids: a review. Annu. Rev. Fluid Mech. 11, 317-338.

McIntyre, M. 2000 On global-scale atmospheric circulations. In Perspectives in Fluid Dynamics (ed. G. Batchelor, H. Moffatt \& M. Worster), pp. 557-624. Cambridge University Press.

Majda, A. \& Grote, M. 1997 Model dynamics and vertical collapse in decaying strongly stratified flows. Phys. Fluids 9, 2932-2940.

MÉtais, O. \& Herring, J. 1989 Numerical simulations of freely evolving turbulence in stably stratified fluids. J. Fluid Mech. 202, 117-148. 
Moulin, F. 2003 Interactions ondes-vortex en milieu stratifié tournant et transport à travers une barrière dynamique. $\mathrm{PhD}$ thesis, Grenoble.

Moulin, F. \& FLór, J. 2004 Vortex wave interaction in a rotating stratified fluid: WKB theory. J. Fluid Mech. Submitted.

Moulin, F. \& Flór, J. 2005 Experimental study on the wave-breaking and mixing properties of an elongated vortex. Dyn. Atmos. Oceans 40,115-130.

Mowbray, D. \& RARITY, B. 1967 A theoretical and experimental investigation of the phase configuration of internal waves of small amplitude in a density stratified liquid. J. Fluid Mech. 28, $1-16$.

Phillips, O. 1966 The Dynamics of the Upper Ocean. Cambridge University Press.

Plougonven, R. \& Zeitlin, V. 2002 Internal gravity wave emission from a pancake vortex: an example of wave-vortex interaction in strongly stratified flows. Phys. Fluids 14, 1259-1268.

Riley, J. \& LelONG, M. 2000 Fluid motions in the presence of strong stable stratification. Annu. Rev. Fluid Mech. 32, 613-657.

Riley, J., Metcalfe, R. \& Weissman, M. 1981 Direct numerical simulations of homogeneous turbulence in density stratified fluids. In Proc. AIP Conf. Nonlinear properties of internal waves (ed. B. West), pp. 79-112. La Jolla Institute.

Spedding, G., Browand, F. \& Fincham, A. 1996 The long-time evolution of the initially-turbulent wake of a sphere in a stable stratification. Dyn. Atmos. Oceans 23, 171-182.

Staquet, C., Bouruet-Aubertot, P. \& Koudella, C. 2001 Mixing by breaking internal gravity waves. In Turbulent Mixing in Geophysical Flows (ed. P. Linden \& J. Redondo), pp. 175-200. CIMNE, Barcelona.

Staquet, C. \& Huerre, G. 2002 On transport accross a barotropic shear flow by breaking inertiagravity waves. Phys. Fluids 14, 1993-2006.

Staquet, C. \& Riley, J. 1989 On the velocity field associated with potential vorticity. Dyn. Atmos. Oceans 14, 93-123.

Staquet, C. \& Sommeria, J. 2002 Internal gravity waves, from instabilities to turbulence. Annu. Rev. Fluid Mech. 34, 559-593.

Sutherland, B. 2000 Internal wave reflection in uniform shear. Q. J. R. Met. Soc. 126, 3255-3286.

Sutherland, B., Dalziel, S., Hughes, G. \& Linden, P. 1999 Visualization and measurement of internal waves by 'synthetic schlieren'. Part 1 . Vertically oscillating cylinder. J. Fluid Mech. 390, 93-126.

ThORPE, S. 1981 An experimental study of critical layers. J. Fluid Mech. 103, 321-344.

Winters, K. \& D'Asaro, E. 1994 Three-dimensional wave breaking near a critical level. J. Fluid Mech. 272, 255-284. 